\title{
Counterintuitive dependence of temporal asymptotics on initial decay in a nonlocal degenerate parabolic equation arising in game theory
}

\author{
Johannes Lankeit* \\ Institut für Mathematik, Universität Paderborn, \\ 33098 Paderborn, Germany
}

\author{
Michael Winkler ${ }^{\dagger}$ \\ Institut für Mathematik, Universität Paderborn, \\ 33098 Paderborn, Germany
}

August 5, 2021

\begin{abstract}
We consider the degenerate parabolic equation with nonlocal source given by

$$
u_{t}=u \Delta u+u \int_{\Omega}|\nabla u|^{2}
$$

which has been proposed as model for the evolution of the density distribution of frequencies with which different strategies are pursued in a population obeying the rules of replicator dynamics in a continuous infinite-dimensional setting.

Firstly, for all positive initial data $u_{0} \in C^{0}\left(\mathbb{R}^{n}\right)$ satisfying $u_{0} \in L^{p}\left(\mathbb{R}^{n}\right)$ for some $p \in(0,1)$ as well as $\int_{\mathbb{R}^{n}} u_{0}=1$, the corresponding Cauchy problem in $\mathbb{R}^{n}$ is seen to possess a global positive classical solution with the property that $\int_{\mathbb{R}^{n}} u(\cdot, t)=1$ for all $t>0$.

Thereafter, the main purpose of this work consists in reavealing a dependence of the large time behavior of these solutions on the spatial decay of the initial data in a direction that seems unexpected when viewed against the background of known behavior in large classes of scalar parabolic problems. In fact, it is shown that all considered solutions asymptotically decay with respect to their spatial $H^{1}$ norm, so that

$$
\mathcal{E}(t):=\int_{0}^{t} \int_{\mathbb{R}^{n}}|\nabla u(\cdot, t)|^{2}, \quad t>0,
$$

always grows in a significantly sublinear manner in that

$$
\frac{\mathcal{E}(t)}{t} \rightarrow 0 \quad \text { as } t \rightarrow \infty
$$

the precise growth rate of $\mathcal{E}$, however, depends on the initial data in such a way that fast decay rates of $u_{0}$ enforce rapid growth of $\mathcal{E}$. To this end, examples of algebraical and certain exponential types of initial decay are detailed, inter alia generating logarithmic and arbitrary sublinear algebraic growth rates of $\mathcal{E}$, and moreover indicating that (0.1) is essentially optimal.
\end{abstract}

Key words: degenerate diffusion; nonlocal source; decay rate

Math Subject Classification (2010): 35B40 (primary), 35K55, 35K65, 91A22 (secondary)

*jlankeit@math.uni-paderborn.de

${ }^{\dagger}$ michael.winkler@math.uni-paderborn.de 


\section{Introduction}

For $n \geq 1$ and given positive initial data $u_{0} \in C^{0}\left(\mathbb{R}^{n}\right)$, we consider positive solutions of the Cauchy problem

$$
\begin{cases}u_{t}=u \Delta u+u \int_{\mathbb{R}^{n}}|\nabla u|^{2}, & x \in \mathbb{R}^{n}, t>0, \\ u(x, 0)=u_{0}(x), & x \in \mathbb{R}^{n},\end{cases}
$$

which has been proposed in the context of evolutionary game theory as a model for strategies which are pursued within a population and the distribution of which evolves in accordance with the rules of replicator dynamics. In this model, the continuum of possible strategies is given by the domain $\mathbb{R}^{n}$ and $u$ plays the role of the density of the relative frequency with which the strategies are followed. The analogue for a setting involving finitely many strategies goes back to the works of Taylor and Jonker [22] and Maynard Smith [17], and infinite-dimensional variants have been treated in [3] and [18]. Building on these, for steep payoff kernels of Gaussian type, in [10, 11] the PDE (1.2) was introduced. For additional details concerning the modeling background, we refer to [9, Appendix A], [11], and the references therein.

Mathematically, (1.2) can be viewed as joining two mechanisms which are quite delicate even when regarded separately: Firstly, the PDE therein contains a reaction term which is superlinear, and hence potentially destabilizing in the extreme sense of possibly enforcing blow-up, and which is moreover nonlocal and thereby may destroy any ordering property, as constituting a well-appreciated feature of parabolic equations with exclusively local terms (cf. the book [21] for a large variety of aspects related to this). Secondly, the diffusion process in (1.2) degenerates near points where $u$ is small, and it is indicated by the analysis of the simple diffusion equation

$$
u_{t}=u^{p} \Delta u
$$

that with regard to this degeneracy, (1.2) is precisely critical: Namely, whereas in the case $p \in(0,1)$ the equation (1.3) actually reduces to a porous medium equation with its well-developed theory on existence, uniqueness and (Hölder) regularity of weak solutions ([1]), it is known that stronger degeneracies in (1.3) may bring about much more irregular and unexpected solution behavior (see e.g. [26] or [27] for two recent examples); the particular borderline situation of the exponent $p=1$ is indicated e.g. by the classical results from [2] and [16]) which assert that precisely for $p \geq 1$, weak solutions to (1.3) can spontaneously develop disconinuities and in general are not unique.

Accordingly, previous studies on the PDE in (1.2) either concentrate on the construction of particular radially symmetric self-similar solutions of self-similar structure, thus actually concerned with a corresponding ODE analysis ([10]), or resort to appropriate generalized solution frameworks, as recently done in [9] for the homogeneous Dirichlet problem associated with (1.2) in bounded domains $\Omega \subset \mathbb{R}^{n}$. Beyond a corresponding local existence statement, the latter work moreover provides some rigorous evidence for the mass evolution property

$$
\frac{d}{d t} \int_{\Omega} u=\left\{\int_{\Omega}|\nabla u|^{2}\right\} \cdot\left\{\int_{\Omega} u-1\right\},
$$

formally satisfied by solutions to the considered problem, in the sense of the implication that if the respective initial data satisfy $\int_{\Omega} u_{0}<1$, a global weak solution $u$ can be found which is such that 
$\int_{\Omega} u(\cdot, t)<1$ for all $t>0$ and such that $u(\cdot, t) \rightarrow 0$ as $t \rightarrow \infty$ in an appropriate sense, while whenever $\int_{\Omega} u_{0}>1$, a solution can be constructed which blows up in finite time $T$ with respect to its spatial $L^{\infty}$ norm, and for which we have $\int_{\Omega} u(\cdot, t)>1$ for all $t \in(0, T)$. For this Dirichlet problem, an essentially complete understanding has also been achieved in the critical case when $\int_{\Omega} u_{0}=1$ : For such initial data, namely, the initial-boundary value problem in question possesses a global generalized solution which satisfies $\int_{\Omega} u(\cdot, t)=1$ for all $t>0$ and furthermore stabilizes toward the solution $\varphi$ of the Dirichlet problem for $-\Delta \varphi=1$ in $\Omega$ in the large time limit ([14]).

Global existence of classical solutions. Bearing in mind that in the present modeling context the unknown $u$ represents a probability density, in this work we shall focus on the analogue of the latter unit-mass situation in the Cauchy problem (1.2), thus concentrating on solutions satisfying the additional condition

$$
\int_{\mathbb{R}^{n}} u(\cdot, t)=1
$$

throughout evolution. Since this in particular requires solutions to attain small values in large spatial regions, even the mere construction of solutions seems to be nontrivial and not to be achievable through a straightforward approximation by solutions to homogeneous Dirichlet problems in balls with increasing size, for instance; in fact, it seems unclear whether such approximate solutions enjoy suitable compactness properties which are sufficient to ensure convergence to a solution of (1.2) additionally satisfying (1.5), rather than e.g. a corresponding inequality only.

We first address this problem of solvability, and it will turn out that despite these obstacles, for a large class of initial data a global solution can actually be found in the classical framework specified as follows.

Definition 1.1. Let $u_{0} \in C^{0}\left(\mathbb{R}^{n}\right)$ be positive. Then by a positive classical solution of (1.2) in $\mathbb{R}^{n} \times$ $(0, \infty)$ we mean a positive function

$$
u \in C^{0}\left(\mathbb{R}^{n} \times[0, \infty)\right) \cap C^{2,1}\left(\mathbb{R}^{n} \times(0, \infty)\right)
$$

which is such that $\nabla u(\cdot, t)$ belongs to $L^{2}\left(\mathbb{R}^{n}\right)$ for all $t>0$, that

$$
(0, \infty) \ni t \mapsto \int_{\mathbb{R}^{n}}|\nabla u(\cdot, t)|^{2} \quad \text { is continuous }
$$

and which satisfies both identities in (1.2) in the pointwise sense.

Within this setting, the first of our main results asserts global solvability under mild assumptions on the spatial decay of $u_{0}$.

Theorem 1.2. Assume that $u_{0} \in C^{0}\left(\mathbb{R}^{n}\right)$ is positive and satisfies

$$
u_{0} \in L^{p}\left(\mathbb{R}^{n}\right) \quad \text { for some } p \in(0,1)
$$

as well as

$$
u_{0}(x) \rightarrow 0 \quad \text { as }|x| \rightarrow \infty
$$

and

$$
\int_{\mathbb{R}^{n}} u_{0}=1
$$


Then the problem (1.2) possesses at least one positive classical solution $u$ in $\mathbb{R}^{n} \times(0, \infty)$ fulfilling

$$
\int_{\mathbb{R}^{n}} u(\cdot, t)=1 \quad \text { for all } t>0 .
$$

Large time behavior. Our next objective consists in deriving some information on the large time behavior of solutions, where it will turn out that a convenient description thereof can be given in terms of the cumulated free energy functional $\mathcal{E}$ given by

$$
\mathcal{E}(t):=\int_{0}^{t} \int_{\mathbb{R}^{n}}|\nabla u|^{2}, \quad t>0
$$

indeed, from (1.2) it may be expected that suitably smooth positive solutions with appropriate spatial decay satisfy the identity

$$
\int_{\mathbb{R}^{n}} \frac{u_{t}^{2}}{u}+\frac{1}{2} \frac{d}{d t} \int_{\mathbb{R}^{n}}|\nabla u|^{2}=\left\{\int_{\mathbb{R}^{n}}|\nabla u|^{2}\right\} \cdot\left\{\frac{d}{d t} \int_{\mathbb{R}^{n}} u\right\}
$$

whence the Dirichlet integral $\int_{\mathbb{R}^{n}}|\nabla u|^{2}$ should in fact play the role of a Lyapunov functional on the set of solutions to (1.2) with conserved unit mass. A rigorous partial verification of this is provided by the following proposition which, beyond a correspondingly expected monotonicty property, asserts decay of this free energy and thereby states a first asymptotic feature of $\mathcal{E}$ which holds without imposing further restrictions on the initial data apart from those in Theorem 1.2 .

Proposition 1.3. Let $u_{0} \in C^{0}\left(\mathbb{R}^{n}\right)$ be positive and such that (1.6)-(1.8) hold, and let $u$ denote the corresponding positive classical solution of (1.2) obtained in Theorem 1.2. Then $(0, \infty) \ni t \mapsto \int_{\Omega}|\nabla u(\cdot, t)|^{2}$ is nonincreasing with

$$
\int_{\mathbb{R}^{n}}|\nabla u(\cdot, t)|^{2} \rightarrow 0 \quad \text { as } t \rightarrow \infty .
$$

In particular, with $\mathcal{E}$ as in (1.10) we have

$$
\frac{\mathcal{E}(t)}{t} \rightarrow 0 \quad \text { as } t \rightarrow \infty
$$

As already suggested by well-known properties of the mere heat equation, but also confirmed in numerous cases of Cauchy problems for nonlinear diffusion equations (see [23] and also [6] and the references therein for some recent developments) and nonlinearly forced semilinear parabolic equations ([15], [5], [21]), more detailed asymptotic properties of solutions may depend on the spatial decay of the initial data in quite a colorful manner. The most commonly found flavor of results in this direction is that in the respective problem a certain monotone dependence, either continuous or dicontinuous, of temporal convergence rates on rates of initially present spatial decay is detected, where in apparently perfect accordance with intuitive ideas, monotonicity is usually directed in such a way that fast spatial decay implies fast temporal decay and vice versa.

In the present context, however, our results indicate that the unit-mass constraint considered here enforces a very subtle balance between nonlocal reaction and nonlinear diffusion in (1.2) which is such that this direction of monotone dependence is actually reversed.

For initial data satisfying algebraic decay conditions consistent with our overall integrability requirements, this becomes manifest in a corresponding counterintuitive ordering of the respective coefficients in logarithmic growth estimates for $\mathcal{E}$ which are the objective of the following two statements. 
Theorem 1.4. Suppose that $u_{0} \in C^{0}\left(\mathbb{R}^{n}\right)$ is positive such that (1.6)-(1.8) are fulfilled, and let $u$ and $\mathcal{E}$ be as in Theorem 1.2 and (1.10).

i) If

$$
u_{0}(x) \geq c_{0}(1+|x|)^{-\gamma} \quad \text { for all } x \in \mathbb{R}^{n}
$$

for some $c_{0}>0$ and $\gamma>n$, then there exist $T>0$ and $C>0$ such that

$$
\mathcal{E}(t) \leq \frac{\gamma-n}{n+2} \ln t+C \quad \text { for all } t>T .
$$

ii) If there exist $C_{0}>0$ and $\gamma>n$ fulfilling

$$
u_{0}(x) \leq C_{0}(1+|x|)^{-\gamma} \quad \text { for all } x \in \mathbb{R}^{n},
$$

then for any $\varepsilon \in(0, \gamma)$ one can find $C(\varepsilon)>0$ with the property that

$$
\mathcal{E}(t) \geq \frac{\gamma-n-\varepsilon}{n+2} \ln t-C(\varepsilon) \quad \text { for all } t>0 .
$$

A natural next problem appears to consist in determining how far it is possible to close the gap between the logarithmic estimates from Theorem 1.4 thus essentially sharp e.g. for initial data with precise algebraic decay, and the general property (1.13) which excludes linear and faster growth of $\mathcal{E}$ but would be well consistent with any sublinearly increasing $\mathcal{E}$. In order to obtain solutions for which $\mathcal{E}$ becomes substantially larger than expressed in Theorem 1.4, we shall consider more rapidly decaying intial data, some prototypical choices of which will be specified below in Corollary 1.6 and Corollary 1.7. For technical reasons, the formulation of our general statement in this direction will require the introduction of a function $\ell \in C^{0}([0, \infty))$ which is such that

$$
\ell(0)=0, \quad \ell>0 \text { in }(0, \infty) \text { and } \quad \ell \text { is nondecreasing on }(0, \infty),
$$

but that $\ell$ grows in a significantly sublinear manner in that

$$
\text { there exists } \xi_{0}>0 \text { such that }\left(\xi_{0}, \infty\right) \ni \xi \mapsto \xi \cdot \ell^{\frac{n+2}{n}}\left(\frac{1}{\xi}\right) \text { is nondecreasing, }
$$

and that

$$
\int_{1}^{\infty} \frac{d \xi}{\xi \cdot \ell^{\frac{n+2}{n}}\left(\frac{1}{\xi}\right)}=\infty
$$

which, for instance, is satisfied for $\ell(\xi):=\xi^{\alpha}, \xi \geq 0$, whenever $\alpha \in\left(0, \frac{n}{n+2}\right)$, but also for $\ell(\xi):=$ $\ln ^{\alpha}(\xi+1), \xi \geq 0$, for arbitrary $\alpha>0$. Given any such $\ell$, we furthermore let

$$
\mathcal{L}(t):=\int_{1}^{t} \frac{d \xi}{\xi \cdot \ell^{\frac{n+2}{n}}\left(\frac{1}{\xi}\right)}, \quad t>1
$$

noting that by (1.17), $\mathcal{L}$ is strictly monotone and so is $\mathcal{L}^{-1}:(0, \infty) \rightarrow(1, \infty)$, which is well-defined due to (1.19). Then under appropriate conditions on the spatial decay of the initial data, the quantity $\mathcal{E}(t)$ can be estimated in terms of $\mathcal{L}$. 
Theorem 1.5. Let $u_{0} \in C^{0}\left(\mathbb{R}^{n}\right)$ be a positive function satisfing (1.6)-(1.8), and let $u$ and $\mathcal{E}$ be as given by Theorem 1.2 and (1.10). Moreover, let $\ell \in C^{0}([0, \infty)$ satisfy (1.17), (1.18) and (1.19).

i) Assume that there exists $\xi_{1} \in(0, \infty]$ such that $\ell$ is strictly increasing on $\left(0, \xi_{1}\right)$, and that

$$
\frac{\xi \ell^{\prime}(\xi)}{\ell(\xi)} \rightarrow 0 \quad \text { as } \xi \searrow 0
$$

Moreover, suppose that there exist $q \in(0,1)$ and $R_{\star}>\max \left\{\frac{1}{\sqrt[n]{\xi_{1}}}, \frac{1}{\sqrt[n]{\lim _{\xi \nearrow \xi_{1}} \ell(\xi)}}\right\}$ such that

$$
u_{0}(x) \geq\left\{\ell^{-1}\left(\frac{1}{|x|^{n}}\right)\right\}^{q} \quad \text { for all } x \in \mathbb{R}^{n} \backslash B_{R_{\star}} .
$$

Then for some $t_{0}>0$ and $C_{1}>0, C_{2} \geq 0$

$$
\mathcal{E}(t) \leq \ln \left(\left(\mathcal{L}^{-1}\right)^{\prime}\left(C_{1} t\right)\right)+C_{2} \quad \text { for all } t>t_{0} .
$$

ii) Suppose that there exist $\xi_{2}>0, a>0$ and $\lambda_{0}>0$ such that $\ell \in C^{2}\left(\left(0, \xi_{2}\right)\right)$ and

$$
\xi \ell^{\prime \prime}(\xi) \geq-\ell^{\prime}(\xi) \quad \text { for all } \xi \in\left(0, \xi_{2}\right)
$$

as well as

$$
\ell(\xi) \leq(1+a \lambda) \ell\left(\xi^{1+\lambda}\right) \quad \text { for all } \xi \in\left(0, \xi_{2}\right) \text { and each } \lambda \in\left(0, \lambda_{0}\right) .
$$

If there exists a radially symmetric $\bar{u}_{0} \in C^{0}\left(\mathbb{R}^{n}\right)$, nonincreasing with respect to $|x|$ and satisfying $u_{0} \leq \bar{u}_{0}$ as well as

$$
\bar{u}_{0}<\min \left\{\xi_{2}^{2}, \xi_{2}^{\frac{2}{1+q_{0}}}\right\} \quad \text { in } \mathbb{R}^{n}
$$

for some $q_{0}>0$ and

$$
\int_{\mathbb{R}^{n}} \ell\left(\bar{u}_{0}\right)<\infty
$$

then we can find $t_{0}>0$ and $C_{1}>0, C_{2} \geq 0$ such that with $\mathcal{E}$ and $\mathcal{L}$ taken from (1.10) and (1.20) we have

$$
\mathcal{E}(t) \geq \ln \left(\left(\mathcal{L}^{-1}\right)^{\prime}\left(C_{1} t\right)\right)-C_{2} \quad \text { for any } t>t_{0}
$$

In particular, arbitrary sublinear algebraic growth of $\mathcal{E}$ occurs for initial data exhibiting certain exponential types of decay. Let us underline that due to the upward monotonicity of the mapping $0<\beta \mapsto \frac{1}{1+\frac{n+2}{n} \beta}$, the following result is again consistent with the above observation that fast spatial decay of $u_{0}$ generates slow decay of the solution.

Corollary 1.6. Let $u_{0} \in C^{0}\left(\mathbb{R}^{n}\right)$ be positive and such that (1.6)-(1.8) hold, and let $u$ and $\mathcal{E}$ be as in Theorem 1.2 and (1.10). 
i) If

$$
u_{0}(x) \geq c_{0} e^{-\alpha|x|^{\beta}} \quad \text { for all } x \in \mathbb{R}^{n} \backslash B_{R_{\star}}
$$

for some $R_{\star}>0, \beta>0, \alpha \in(0,1)$ and $c_{0}>0$, then there exist $t_{0}>0$ and $C>0$ such that

$$
\mathcal{E}(t) \leq C t^{1 /\left(1+\frac{n+2}{\beta}\right)} \quad \text { for all } t>t_{0} .
$$

ii) If $u_{0}$ satisfies

$$
u_{0}(x) \leq C_{0} e^{-\alpha|x|^{\beta}}
$$

for some $C_{0}>0, \alpha>0$ and $\beta>0$, then for any $\varepsilon>0$ we can find $t_{0}>0$ and $c>0$ such that

$$
\mathcal{E}(t) \geq c t^{1 /\left(1+\frac{n+2}{\beta}+\varepsilon\right)} \quad \text { for all } t>t_{0} .
$$

But also quite tiny deviations from linear growth of $\mathcal{E}$ can be enforced by appropriate choices of the initial data. This is indicated by a second consequence of Theorem 1.5 on a corresponding logarithmic correction for very rapidly decaying data.

Corollary 1.7. Let $u_{0} \in C^{0}\left(\mathbb{R}^{n}\right)$ be a positive function, satisfying (1.6)-(1.8) as well as

$$
u_{0}(x) \leq C_{0} e^{-\alpha e^{|x|^{\beta}}} \quad \text { for all } x \in \mathbb{R}^{n}
$$

with some positive constants $C_{0}>0, \alpha>0$ and $\beta>0$. Then for any $\varepsilon>0$ there exist $t_{0}>0$ and $c>0$ such that with $u$ and $\mathcal{E}$ taken from Theorem 1.2 and (1.10), we have

$$
\mathcal{E}(t) \geq c t(\ln t)^{-\left(\frac{n+2}{\beta}+\varepsilon\right)} \quad \text { for all } t>t_{0} .
$$

Plan of the paper. A key for our analysis consists in the observation that if for a supposedly given classical solution of (1.2) we let $L(t):=\int_{\mathbb{R}^{n}}|\nabla u(\cdot, t)|^{2}, t>0$, then upon suitable choices of the functions $f$ and $H$ on $[0, \infty)$, the substitution $v(s, t):=f(s) u(x, t)$ with $t=H(s)$ transforms the equation $u_{t}=u \Delta u+L(t) u$ satisfied by $u$ to the yet degenerate but local unforced diffusion equation

$$
v_{s}=v \Delta v \text {. }
$$

Accordingly, as a prerequisite for our existence proof we will first make sure that under essentially the same assumptions on the initial data as in Theorem 1.2, the Cauchy problem for the latter equation possesses a smooth positive solution enjoying certain favorable spatial decay properties which inter alia allow for a precise control of the respective mass evolution as well as for the important conclusion that the corresponding Dirichlet integral depends continuously on the time variable (see Section 2 and especially Lemma 2.5 and Lemma 2.7). Relying on a known fundamental asymptotic property of these solutions (Lemma 3.2), in Section 3 it will thereupon be possible to construct a suitable substitution which indeed transforms solutions of (1.24) into classical solutions of (1.2) with conserved unit mass and some additional regularity properties (Lemma 3.5), the latter enabling us to justify the monotonicty property suggested by (1.11) and thereby establish Proposition 1.3 in Section 4 , On the basis of known refined temporal decay estimates for solutions to (1.24), in Section 5 we will obtain Theorem 1.4 as an immediate consequence of the literature, whereas in Section 6 a more detailed analysis will be performed so as to finally yield the inequalities claimed in Theorem 1.5. Corollary 1.6] and Corollary 1.7 . 


\section{Analysis of the Cauchy problem for $v_{s}=v \Delta v$}

In this section we consider the corresponding initial-value problem

$$
\begin{cases}v_{s}=v \Delta v, & x \in \mathbb{R}^{n}, s>0, \\ v(x, 0)=v_{0}(x), & x \in \mathbb{R}^{n},\end{cases}
$$

with given positive data $v_{0} \in L^{\infty}\left(\mathbb{R}^{n}\right) \cap C^{0}\left(\mathbb{R}^{n}\right)$, and seek for a solution to the original problem by an adequate change of variables. This will be achieved in Lemma 3.5 on the basis of the following result on classical solvability of (2.1) under these mild hypotheses on the initial data, as obtained in [7. Proposition 1.1].

Lemma 2.1. Suppose that $v_{0} \in L^{\infty}\left(\mathbb{R}^{n}\right) \cap C^{0}\left(\mathbb{R}^{n}\right)$ is positive. Then the problem (2.1) possesses at least one global classical solution $v \in C^{0}\left(\mathbb{R}^{n} \times[0, \infty)\right) \cap C^{2,1}\left(\mathbb{R}^{n} \times(0, \infty)\right)$ which satisfies

$$
0<v(x, s) \leq\left\|v_{0}\right\|_{L^{\infty}\left(\mathbb{R}^{n}\right)} \quad \text { for all } x \in \mathbb{R}^{n} \text { and } s \geq 0 .
$$

Moreover, this solution is minimal in the sense that whenever $T \in(0, \infty]$ and $\widetilde{v} \in C^{0}\left(\mathbb{R}^{n} \times[0, T)\right) \cap$ $C^{2,1}\left(\mathbb{R}^{n} \times(0, T)\right)$ are such that $\widetilde{v}$ is positive and solves (2.1) classically in $\mathbb{R}^{n} \times(0, T)$, we necessarily have $v \leq \widetilde{v}$ in $\mathbb{R}^{n} \times(0, T)$.

A convenient additional property of the minimal solution gained above is that it can be approximated by classical solutions of certain regularized problems in bounded domains. To make this more precise, throughout the sequel abbreviating $B_{R}:=B_{R}(0)$, let us fix a family $\left(v_{0 R}\right)_{R>0} \subset C^{3}\left(\bar{B}_{R}\right)$ of functions satisfying $0<v_{0 R}<v_{0}$ in $B_{R}$ and $v_{0 R}=0$ on $\partial B_{R}$ as well as $v_{0 R} \nearrow v_{0}$ in $\mathbb{R}^{n}$ as $R \nearrow \infty$, and consider the Dirichlet problems

$$
\begin{cases}v_{R s}=v_{R} \Delta v_{R}, & x \in B_{R}, s>0, \\ v_{R}(x, s)=0, & x \in \partial B_{R}, s>0, \\ v_{R}(x, 0)=v_{0 R}(x), & x \in B_{R},\end{cases}
$$

for $R>0$, along with the non-degenerate approximate versions thereof given by

$$
\begin{cases}v_{R \varepsilon s}=v_{R \varepsilon} \Delta v_{R \varepsilon}, & x \in B_{R}, s>0, \\ v_{R \varepsilon}(x, t)=\varepsilon, & x \in \partial B_{R}, s>0, \\ v_{R \varepsilon}(x, 0)=v_{0 R \varepsilon}(x), & x \in B_{R},\end{cases}
$$

for $\varepsilon \in(0,1)$, where we have set

$$
v_{0 R \varepsilon}:=v_{0 R}+\varepsilon .
$$

We then indeed have the following ([7, Lemma 2.1]):

Lemma 2.2. Suppose that $v_{0} \in L^{\infty}\left(\mathbb{R}^{n}\right) \cap C^{0}\left(\mathbb{R}^{n}\right)$ is positive. Then with $v_{0 R}$ and $v_{0 \varepsilon R}$ as above, any of the problems (2.3) possesses a global classical solution $v_{R \varepsilon} \in C^{0}\left(\bar{B}_{R} \times[0, \infty)\right) \cap C^{2,1}\left(\bar{B}_{R} \times(0, \infty)\right)$. As $\varepsilon \searrow 0$, these solutions satisfy $v_{R \varepsilon} \searrow v_{R}$ with a positive classical solution $v_{R} \in C^{0}\left(\bar{B}_{R} \times[0, \infty)\right) \cap$ $C^{2,1}\left(B_{R} \times(0, \infty)\right)$ of (2.2), and moreover we have

$$
v_{R} \nearrow v \text { in } \mathbb{R}^{n} \times(0, \infty) \quad \text { as } R \nearrow \infty,
$$

where $v$ denotes the minimal classical solution of (2.1) addressed in Lemma 2.1] 
For later reference, let us note that in the special case when $v_{0}$ is radially symmetric around the origin and nonincreasing with respect to $|x|$, we may assume that $v_{0 R}$ has the same properties. According to a standard argument based on the comparison principle applied to (2.3), this entails that in this situation also $v_{R \varepsilon}(\cdot, s)$ and $v_{R}(\cdot, s)$ are radially symmetric and nonincreasing with respect to $|x| \in[0, R]$ for each $s>0$.

\subsection{Temporally uniform spatial decay}

The goal of this section is to make sure that the minimal solution of (2.1) will inherit an initially present spatial decay in the following temporally uniform sense:

Lemma 2.3. Suppose that $v_{0} \in C^{0}\left(\mathbb{R}^{n}\right)$ is positive and such that

$$
v_{0}(x) \rightarrow 0 \quad \text { as }|x| \rightarrow \infty .
$$

Then the minimal solution $v$ of (2.1) satisfies $v(\cdot, s) \rightarrow 0$ as $|x| \rightarrow \infty$, uniformly with respect to $s \geq 0$; that is, we have

$$
\|v\|_{L^{\infty}\left(\left(\mathbb{R}^{n} \backslash B_{R}\right) \times(0, \infty)\right)} \rightarrow 0 \quad \text { as } R \rightarrow \infty .
$$

In order to prepare a proof of this, let us recall the following implication of (2.4) on the large time behavior of $v$, as derived in [25, Thm. 3.1].

Lemma 2.4. Assume that the positive function $v_{0} \in C^{0}\left(\mathbb{R}^{n}\right)$ satisfies $v_{0}(x) \rightarrow 0$ as $|x| \rightarrow \infty$. Then the minimal solution $v$ of (2.1) has the property that

$$
\|v(\cdot, s)\|_{L^{\infty}\left(\mathbb{R}^{n}\right)} \rightarrow 0 \quad \text { as } s \rightarrow \infty .
$$

We will rely on this lemma in the following

Proof of Lemma 2.3. For $x \in \mathbb{R}^{n}$ replacing $v_{0}(x)$ with $\bar{v}_{0}(x):=\left\|v_{0}\right\|_{L^{\infty}\left(\mathbb{R}^{n} \backslash B_{|x|}\right)}$ if necessary, on recalling the comment following Lemma 2.2 we may assume that $v_{0}$ is radially symmetric and nonincreasing with respect to $|x| \geq 0$, and that for each $R>0$ also $v_{0 R}$ and $v_{R}(\cdot, s)$ are radial and nonincreasing in $|x| \in[0, R]$ for $s>0$.

Now if (2.5) was false, there would exist $\delta_{0}>0,\left(R_{k}\right)_{k \in \mathbb{N}} \subset(0, \infty)$ and $\left(s_{k}\right)_{k \in \mathbb{N}} \subset(0, \infty)$ such that $R_{k} \rightarrow \infty$ as $k \rightarrow \infty$ and

$$
\left\|v\left(\cdot, s_{k}\right)\right\|_{L^{\infty}\left(\mathbb{R}^{n} \backslash B_{R_{k}}\right)} \geq \delta_{0} \quad \text { for all } k \in \mathbb{N} .
$$

Here in the case when $s_{k} \rightarrow \infty$ as $k \rightarrow \infty$, invoking Lemma 2.4 we would see that

$$
\delta_{0} \leq\left\|v\left(\cdot, s_{k}\right)\right\|_{L^{\infty}\left(\mathbb{R}^{n} \backslash B_{R_{k}}\right)} \leq\left\|v\left(\cdot, s_{k}\right)\right\|_{L^{\infty}\left(\mathbb{R}^{n}\right)} \rightarrow 0 \quad \text { as } k \rightarrow \infty
$$

which is absurd. We are thus left with the case when $\left(s_{k}\right)_{k \in \mathbb{N}}$ has a finite point of accumulation, in which on passing to a subsequence we may assume that there exists $s_{2} \in[0, \infty)$ such that as $k \rightarrow \infty$ we have $s_{k} \rightarrow s_{2}$. We first claim that then

$$
v\left(\cdot, s_{2}\right) \geq \delta_{0} \quad \text { in } \mathbb{R}^{n}
$$


In fact, given any $x_{0} \in \mathbb{R}^{n}$ we may use that $R_{k} \rightarrow \infty$ as $k \rightarrow \infty$ to fix $k_{0} \in \mathbb{N}$ such that $R_{k} \geq\left|x_{0}\right|$ for all $k \geq k_{0}$. As $v\left(\cdot, s_{k}\right)$ is nonincreasing with respect to $|x| \geq 0$, we thus obtain that

$$
v\left(x_{0}, s_{k}\right) \geq\left\|v\left(\cdot, s_{k}\right)\right\|_{L^{\infty}\left(\mathbb{R}^{n} \backslash B_{R_{k}}\right)} \geq \delta_{0} \quad \text { for all } k \geq k_{0},
$$

and that since by continuity of $v$ we have $v\left(x_{0}, s_{k}\right) \rightarrow v\left(x_{0}, s_{2}\right)$ as $k \rightarrow \infty$, therefore indeed $v\left(x_{0}, s_{2}\right) \geq$ $\delta_{0}$.

Having thereby verified (2.6), we proceed to show that actually

$$
v \geq \delta_{0} \quad \text { in } \mathbb{R}^{n} \times\left(s_{2}, \infty\right) .
$$

To see this, for fixed $x_{0} \in \mathbb{R}^{n}$ and $R>0$ we let $\varphi_{R}$ and $\lambda_{R}$ denote the first Dirichlet eigenfunction of $-\Delta$ in $B_{R}\left(x_{0}\right)$, normalized such that $\max _{x \in \bar{B}_{R}\left(x_{0}\right)} \varphi_{R}(x)=\varphi_{R}\left(x_{0}\right)=1$, and the associated principal eigenvalue $\lambda_{R}>0$. For $\delta \in\left(0, \delta_{0}\right)$, we then define $y_{R}$ to be the solution of the initial-value problem

$$
\left\{\begin{array}{l}
y_{R}^{\prime}(s)=-\lambda_{R} y_{R}^{2}(s), \quad s>s_{2} \\
y_{R}\left(s_{2}\right)=\delta
\end{array}\right.
$$

that is, we let

$$
y_{R}(s):=\frac{\delta}{1+\lambda_{R} \delta\left(s-s_{2}\right)}, \quad s \geq s_{2}
$$

and thereafter we introduce a comparison function $\underline{v}$ by writing

$$
\underline{v}(x, s):=y_{R}(s) \varphi_{R}(x), \quad x \in \bar{B}_{R}\left(x_{0}\right), s \geq s_{2} .
$$

Then clearly $\underline{v}(x, s)=0<v(x, s)$ for all $x \in \partial B_{R}\left(x_{0}\right)$ and $s \geq s_{2}$, whereas (2.6) ensures that also $\underline{v}\left(x, s_{2}\right) \leq y_{R}\left(s_{2}\right)=\delta<v\left(x, s_{2}\right)$ for all $x \in \bar{B}_{R}\left(x_{0}\right)$. As furthermore the definition of $\varphi_{R}$ and (2.8) guarantee that

$$
\begin{aligned}
\underline{v}_{s}-\underline{v} \Delta \underline{v} & =y_{R}^{\prime} \varphi_{R}-y_{R}^{2} \varphi_{R} \Delta \varphi_{R} \\
& =y_{R}^{\prime} \varphi_{R}+\lambda_{R} y_{R}^{2} \varphi_{R}^{2} \\
& \leq y_{R}^{\prime} \varphi_{R}+\lambda_{R} y_{R}^{2} \varphi_{R} \\
& =0 \quad \text { in } B_{R}\left(x_{0}\right) \times\left(s_{2}, \infty\right),
\end{aligned}
$$

an appropriate comparison principle $([24])$ shows that $v \geq \underline{v}$ in $\bar{B}_{R}\left(x_{0}\right) \times\left[s_{2}, \infty\right)$, whence in particular

$$
v\left(x_{0}, s\right) \geq \underline{v}\left(x_{0}, s\right)=y_{R}(s) \varphi_{R}\left(x_{0}\right)=\frac{\delta}{1+\lambda_{R} \delta\left(s-s_{2}\right)} \quad \text { for all } s>s_{2} .
$$

Taking $\delta \nearrow \delta_{0}$ and using that $\lambda_{R} \rightarrow 0$ as $R \rightarrow \infty$, we thereby readily obtain that $v\left(x_{0}, s\right) \geq \delta_{0}$ for all $s>s_{2}$. This establishes (2.7) and therefore evidently contradicts the outcome of Lemma 2.4 also in this case. 


\subsection{Time evolution of $L^{p}$ seminorms}

For arbitrary $p>0$, let us next provide a rigorous counterpart of the identity

$$
\frac{d}{d s} \int_{\mathbb{R}^{n}} v^{p}=-p^{2} \int_{\mathbb{R}^{n}} v^{p-1}|\nabla v|^{2},
$$

as formally obtained on testing (2.1) by $v^{p-1}$, which we state in a form that avoids time derivatives which due to the lack of appropriate regularity assumptions on $v$ might not exist. The correspondingly obtained result (2.9) will frequently be applied in the sequel, inter alia to the value $p:=1$ for establishing the desired mass conservation property of our solution $u$ to the original problem (1.2), but also e.g. to $p:=2$ (Lemmata 2.6, 3.5) and to certain $p \in(0,1)$ (Lemma 2.7).

Lemma 2.5. Assume that with some $p>0$, the positive function $v_{0}$ belongs to $L^{p}\left(\mathbb{R}^{n}\right) \cap L^{\infty}\left(\mathbb{R}^{n}\right) \cap$ $C^{0}\left(\mathbb{R}^{n}\right)$, and suppose that $v$ is a bounded positive classical solution of (2.1) in $\mathbb{R}^{n} \times(0, T)$ for some $T \in(0, \infty]$. Then $v(\cdot, s) \in L^{p}\left(\mathbb{R}^{n}\right)$ for all $s \in(0, T)$ and $v^{p-1}|\nabla v|^{2} \in L^{1}\left(\mathbb{R}^{n} \times(0, T)\right)$, and moreover we have the identity

$$
\int_{\mathbb{R}^{n}} v^{p}(\cdot, s)+p^{2} \int_{0}^{s} \int_{\mathbb{R}^{n}} v^{p-1}|\nabla v|^{2}=\int_{\mathbb{R}^{n}} v_{0}^{p} \quad \text { for all } s \in(0, T) .
$$

Proof. We first claim that

$$
\int_{\mathbb{R}^{n}} v^{p}(\cdot, s)+p^{2} \int_{0}^{s} \int_{\mathbb{R}^{n}} v^{p-1}|\nabla v|^{2} \leq \int_{\mathbb{R}^{n}} v_{0}^{p} \quad \text { for all } s \in(0, T) .
$$

To establish this inequality, given $\alpha>0$ and $R>0$ we introduce $\varphi_{\alpha}(x):=e^{-\alpha|x|}$ for $x \in \mathbb{R}^{n}$ as well as

$$
\zeta_{R}(x):= \begin{cases}1 & \text { if } x \in B_{R}, \\ R+1-|x| & \text { if } x \in B_{R+1} \backslash B_{R}, \\ 0 & \text { if } x \in \mathbb{R}^{n} \backslash B_{R+1},\end{cases}
$$

and note that then both $\varphi_{\alpha}$ and $\zeta_{R}$ belong to $W^{1, \infty}\left(\mathbb{R}^{n}\right)$. By positivity of $v$, we may thus test (2.1) against $\zeta_{R}^{2} \varphi_{\alpha} v^{p-1}$ to see that since $\zeta_{R}$ has compact support in $\mathbb{R}^{n}$, we have

$$
\frac{d}{d s} \int_{\mathbb{R}^{n}} \zeta_{R}^{2} \varphi_{\alpha} v^{p}+p^{2} \int_{\mathbb{R}^{n}} \zeta_{R}^{2} \varphi_{\alpha} v^{p-1}|\nabla v|^{2}=-p \int_{\mathbb{R}^{n}} v^{p} \nabla v \cdot \nabla\left(\zeta_{R}^{2} \varphi_{\alpha}\right) \quad \text { for all } s \in(0, T) .
$$

For fixed $\eta \in(0,1)$, by means of Young's inequality we find that herein

$$
\left|-p \int_{\mathbb{R}^{n}} v^{p} \nabla v \cdot \nabla\left(\zeta_{R}^{2} \varphi_{\alpha}\right)\right| \leq \eta p^{2} \int_{\mathbb{R}^{n}} \zeta_{R}^{2} \varphi_{\alpha} v^{p-1}|\nabla v|^{2}+\frac{1}{4 \eta} \int_{\mathbb{R}^{n}} \frac{\left|\nabla\left(\zeta_{R}^{2} \varphi_{\alpha}\right)\right|^{2}}{\zeta_{R}^{2} \varphi_{\alpha}} \cdot v^{p+1} \quad \text { for all } s \in(0, T),
$$

where we can estimate

$$
\begin{aligned}
\left|\nabla\left(\zeta_{R}^{2} \varphi_{\alpha}\right)\right|^{2} & =\left|2 \zeta_{R} \varphi_{\alpha} \nabla \zeta_{R}+\zeta_{R}^{2} \nabla \varphi_{\alpha}\right|^{2} \\
& \leq 8 \zeta_{R}^{2} \varphi_{\alpha}^{2}\left|\nabla \zeta_{R}\right|^{2}+2 \zeta_{R}^{4}\left|\nabla \varphi_{\alpha}\right|^{2} \quad \text { a.e. in } \mathbb{R}^{n}
\end{aligned}
$$


and hence

$$
\frac{1}{4 \eta} \int_{\mathbb{R}^{n}} \frac{\left|\nabla\left(\zeta_{R}^{2} \varphi_{\alpha}\right)\right|^{2}}{\zeta_{R}^{2} \varphi_{\alpha}} \cdot v^{p+1} \leq \frac{2}{\eta} \int_{\Omega}\left|\nabla \zeta_{R}\right|^{2} \varphi_{\alpha} v^{p+1}+\frac{1}{2 \eta} \int_{\Omega} \zeta_{R}^{2} \cdot \frac{\left|\nabla \varphi_{\alpha}\right|^{2}}{\varphi_{\alpha}} \cdot v^{p+1} \quad \text { for all } s \in(0, T) .
$$

Recalling that $M:=\|v\|_{L^{\infty}\left(\mathbb{R}^{n} \times(0, T)\right)}$ is finite by assumption, since $\left|\nabla \zeta_{R}\right| \leq 1$ a.e. in $\mathbb{R}^{n}$ and $\operatorname{supp} \nabla \zeta_{R} \subset$ $\bar{B}_{R+1} \backslash B_{R}$ we see that

$$
\frac{2}{\eta} \int_{\Omega}\left|\nabla \zeta_{R}\right|^{2} \varphi_{\alpha} v^{p+1} \leq \delta_{\eta \alpha R}:=\frac{2 M^{p+1}}{\eta} \int_{B_{R+1} \backslash B_{R}} \varphi_{\alpha} \quad \text { for all } s \in(0, T),
$$

where evidently for each fixed $\eta \in(0,1)$ and $\alpha>0$ we have

$$
\delta_{\eta \alpha R} \rightarrow 0 \quad \text { as } R \rightarrow \infty .
$$

In estimating the second summand on the right of (2.14) we use that $\frac{\left|\nabla \varphi_{\alpha}\right|^{2}}{\varphi_{\alpha}^{2}}=\alpha^{2}$ in $\mathbb{R}^{n} \backslash\{0\}$ to obtain

$$
\frac{1}{2 \eta} \int_{\Omega} \zeta_{R}^{2} \cdot \frac{\left|\nabla \varphi_{\alpha}\right|^{2}}{\varphi_{\alpha}} \cdot v^{p+1} \leq \frac{M \alpha^{2}}{2 \eta} \int_{\mathbb{R}^{n}} \zeta_{R}^{2} \varphi_{\alpha} v^{p} \quad \text { for all } s \in(0, T)
$$

Combined with (2.11)-(2.15), this shows that for $y_{\alpha R}(s):=\int_{\mathbb{R}^{n}} \zeta_{R}^{2} \varphi_{\alpha} v^{p}(\cdot, s), s \in[0, T)$, and $f_{\alpha R}(s):=$ $p^{2} \int_{\mathbb{R}^{n}} \zeta_{R}^{2} \varphi_{\alpha} v^{p-1}(\cdot, s)|\nabla v(\cdot, s)|^{2}, s \in(0, T)$, writing $c_{1}:=\frac{M}{2 \eta}$ we have

$$
y_{\alpha R}^{\prime}(s)+(1-\eta) f_{\alpha R}(s) \leq c_{1} \alpha^{2} y_{\alpha R}(s)+\delta_{\eta \alpha R} \quad \text { for all } s \in(0, T),
$$

which on integration yields

$$
\begin{aligned}
e^{-c_{1} \alpha^{2} s} y_{\alpha R}(s)+(1-\eta) \int_{0}^{s} e^{-c_{1} \alpha^{2} \sigma} f_{\alpha R}(\sigma) d \sigma & \leq y_{\alpha R}(0)+\delta_{\eta \alpha R} \int_{0}^{s} e^{-c_{1} \alpha^{2} \sigma} d \sigma \\
& \leq y_{\alpha R}(0)+\frac{\delta_{\eta \alpha R}}{c_{1} \alpha^{2}} \quad \text { for all } s \in(0, T) .
\end{aligned}
$$

We now observe that since $\zeta_{R} \nearrow 1$ in $\mathbb{R}^{n}$ as $R \nearrow \infty$, Beppo Levi's theorem asserts that for each $s \in(0, T)$,

$$
y_{\alpha R}(s) \nearrow y_{\alpha}(s):=\int_{\mathbb{R}^{n}} \varphi_{\alpha} v^{p}(\cdot, s) \quad \text { as } R \nearrow \infty,
$$

and that for all $s \in(0, T)$ we have

$$
f_{\alpha R}(s) \nearrow f_{\alpha}(s):=p^{2} \int_{\mathbb{R}^{n}} \varphi_{\alpha} v^{p-1}(\cdot, s)|\nabla v(\cdot, s)|^{2} \quad \text { as } R \nearrow \infty .
$$

According to (2.16), from (2.19) we thus infer that

$$
e^{-c_{1} \alpha^{2} s} y_{\alpha}(s)+(1-\eta) \int_{0}^{s} e^{-c_{1} \alpha^{2} \sigma} f_{\alpha}(\sigma) d \sigma \leq y_{\alpha}(0) \quad \text { for all } s \in(0, T) .
$$

As a similar argument based on monotone convergence warrants that for $s \in[0, T)$ we have

$$
y_{\alpha}(s) \nearrow y(s):=\int_{\mathbb{R}^{n}} v^{p}(\cdot, s) \quad \text { as } \alpha \searrow 0
$$


and that

$$
f_{\alpha}(s) \nearrow f(s):=p^{2} \int_{\mathbb{R}^{n}} v^{p-1}(\cdot, s)|\nabla v(\cdot, s)|^{2} \quad \text { as } \alpha \searrow 0
$$

for each $s \in(0, T)$, once more by Beppo Levi's theorem we conclude from (2.22) that

$$
y(s)+(1-\eta) \int_{0}^{s} f(\sigma) d \sigma \leq y(0) \quad \text { for all } s \in(0, T) .
$$

which proves (2.10), because $\eta \in(0,1)$ was arbitrary.

In order to show that conversely also

$$
\int_{\mathbb{R}^{n}} v^{p}(\cdot, s)+p^{2} \int_{0}^{s} \int_{\mathbb{R}^{n}} v^{p-1}|\nabla v|^{2} \geq \int_{\mathbb{R}^{n}} v_{0}^{p} \quad \text { for all } s \in(0, T),
$$

given $\eta \in(0,1)$ we again combine (2.11) with (2.12)-(2.15) and (2.17) to see that

$$
y_{\alpha R}^{\prime}(s)+(1+\eta) f_{\alpha R}(s) \geq-c_{1} \alpha^{2} y_{\alpha R}(s)-\delta_{\eta \alpha R} \quad \text { for all } s \in(0, T),
$$

and that hence

$$
e^{c_{1} \alpha^{2} s} y_{\alpha R}(s)+(1+\eta) \int_{0}^{s} e^{c_{1} \alpha^{2} \sigma} f_{\alpha R}(\sigma) d \sigma \geq y_{\alpha R}(0)-\frac{\delta_{\eta \alpha R}}{c_{1} \alpha^{2}} \quad \text { for all } s \in(0, T) .
$$

On taking $R \searrow \infty$, by (2.16), (2.20) and (2.21) we thereby obtain that

$$
e^{c_{1} \alpha^{2} s} y_{\alpha}(s)+(1+\eta) \int_{0}^{s} e^{+c_{1} \alpha^{2} \sigma} f_{\alpha}(\sigma) d \sigma \geq y_{\alpha}(0) \quad \text { for all } s \in(0, T),
$$

where in passing to the limit in the integral involving $f_{\alpha R}$ we have used the dominated convergence theorem along with the inequality

$$
e^{c_{1} \alpha^{2} \sigma} f_{\alpha R}(\sigma) \leq e^{c_{1} \alpha^{2} s} f_{\alpha}(\sigma)
$$

valid for all $\sigma \in(0, s)$ by (2.21), and the fact that $f_{\alpha}$ is integrable over $(0, s)$ due to e.g. (2.25) and (2.24), for any $s \in(0, T)$. Likewise, one more application of the dominated convergence theorem on the basis of (2.23) and (2.24) finally shows that (2.27) entails the inequality

$$
y(s)+(1+\eta) \int_{0}^{s} f(\sigma) d \sigma \geq y(0) \quad \text { for all } s \in(0, T),
$$

and thereby yields (2.26) on letting $\eta \searrow 0$.

\subsection{Time continuity and integrability of $\int_{\mathbb{R}^{n}}|\nabla v|^{2}$}

In view of the regularity requirements in Definition 1.1 an inevitable task consists in showing continuous dependence of the Dirichlet integral in (1.2) on the time variable. In the transformed problem, this amounts to showing that $0 \leq s \mapsto \int_{\mathbb{R}^{n}}|\nabla v(\cdot, s)|^{2}$ is continuous, which is prepared by the following statement on a corresponding temporally uniform spatial decay property that will beyond this also be useful in Lemma 3.5 and Proposition 1.3 below. 
Lemma 2.6. Suppose that $v_{0} \in L^{2}\left(\mathbb{R}^{n}\right) \cap L^{\infty}\left(\mathbb{R}^{n}\right) \cap C^{0}\left(\mathbb{R}^{n}\right)$ is positive, and that with some $s_{2}>0$ and $T \in\left(s_{2}, \infty\right], v$ is a bounded positive classical solution of (2.1) in $\mathbb{R}^{n} \times(0, T)$ which is such that $v\left(\cdot, s_{2}\right)$ belongs to $W^{1,2}\left(\mathbb{R}^{n}\right)$. Then $v(\cdot, s) \in W^{1,2}\left(\mathbb{R}^{n}\right)$ for all $s \in\left(s_{2}, T\right)$ and $v|\Delta v|^{2} \in L^{1}\left(\mathbb{R}^{n} \times\left(s_{2}, T\right)\right)$, and for each $R>0$ we have

$$
\begin{aligned}
\int_{\mathbb{R}^{n} \backslash B_{R+1}}|\nabla v(\cdot, s)|^{2}+\int_{s_{2}}^{s} \int_{\mathbb{R}^{n} \backslash B_{R+1}} v|\Delta v|^{2} \leq & \int_{\mathbb{R}^{n} \backslash B_{R}}\left|\nabla v\left(\cdot, s_{2}\right)\right|^{2} \\
& +4 \int_{s_{2}}^{s} \int_{B_{R+1} \backslash B_{R}} v|\nabla v|^{2} \quad \text { for all } s \in\left(s_{2}, T\right) .
\end{aligned}
$$

Proof. We fix $R>0$ and let $\zeta_{\rho} \in W^{1, \infty}\left(\mathbb{R}^{n}\right)$ be defined by

$$
\zeta_{\rho}(x):= \begin{cases}0, & x \in B_{R}, \\ |x|-R, & x \in B_{R+1} \backslash B_{R}, \\ 1, & x \in B_{\rho} \backslash B_{R+1}, \\ \rho+1-|x|, & x \in B_{\rho+1} \backslash B_{\rho}, \\ 0, & x \in \mathbb{R}^{n} \backslash B_{\rho+1},\end{cases}
$$

for $\rho>R+1$. Then since $v$ is smooth in $\mathbb{R}^{n} \times(0, T)$, we may use (2.1) to compute

$$
\begin{aligned}
\frac{1}{2} \int_{\mathbb{R}^{n}} \zeta_{\rho}^{2}|\nabla v(\cdot, s)|^{2}-\frac{1}{2} \int_{\mathbb{R}^{n}} \zeta_{\rho}^{2}\left|\nabla v\left(\cdot, s_{2}\right)\right|^{2} & =-\int_{s_{2}}^{s} \int_{\mathbb{R}^{n}} \zeta_{\rho}^{2} v_{s} \Delta v-2 \int_{s_{2}}^{s} \int_{\mathbb{R}^{n}} \zeta_{\rho} v_{s}\left(\nabla v \cdot \nabla \zeta_{\rho}\right) \\
& =-\int_{s_{2}}^{s} \int_{\mathbb{R}^{n}} \zeta_{\rho}^{2} v|\Delta v|^{2}-2 \int_{s_{2}}^{s} \int_{\mathbb{R}^{n}} \zeta_{\rho} v \Delta v\left(\nabla v \cdot \nabla \zeta_{\rho}\right)
\end{aligned}
$$

for all $s \in\left(s_{2}, T\right)$, where by Young's inequality,

$$
\begin{aligned}
-2 \int_{s_{2}}^{s} \int_{\mathbb{R}^{n}} \zeta_{\rho} v \Delta v\left(\nabla v \cdot \nabla \zeta_{\rho}\right) \leq & \frac{1}{2} \int_{s_{2}}^{s} \int_{\mathbb{R}^{n}} \zeta_{\rho}^{2} v|\Delta v|^{2}+2 \int_{s_{2}}^{s} \int_{\mathbb{R}^{n}}\left|\nabla \zeta_{\rho}\right|^{2} v|\nabla v|^{2} \\
\leq & \frac{1}{2} \int_{s_{2}}^{s} \int_{\mathbb{R}^{n}} \zeta_{\rho}^{2} v|\Delta v|^{2}+2 \int_{s_{2}}^{s} \int_{B_{R+1} \backslash B_{R}} v|\nabla v|^{2} \\
& +2 \int_{s_{2}}^{s} \int_{B_{\rho+1} \backslash B_{\rho}} v|\nabla v|^{2} \quad \text { for all } s \in\left(s_{2}, T\right),
\end{aligned}
$$

because $\left|\nabla \zeta_{\rho}\right| \leq 1$ a.e. in $\mathbb{R}^{n}$ and $\nabla \zeta_{\rho}=0$ outside $\left(B_{R+1} \backslash B_{R}\right) \cup\left(B_{\rho+1} \backslash B_{\rho}\right)$. Here we note that our assumptions on $v_{0}$ and $v$ together with the outcome of Lemma 2.5, applied to $p:=2$, ensure that $v|\nabla v|^{2} \in L^{1}\left(\mathbb{R}^{n} \times(0, T)\right)$ and hence

$$
\int_{s_{2}}^{s} \int_{B_{\rho+1} \backslash B_{\rho}} v|\nabla v|^{2} \rightarrow 0 \quad \text { as } \rho \rightarrow \infty
$$

by the dominated convergence theorem. Since as $\rho \rightarrow \infty$ we furthermore have

$$
\zeta_{\rho}(x) \nearrow \zeta(x):= \begin{cases}0, & x \in B_{R}, \\ |x|-R, & x \in B_{R+1} \backslash B_{R}, \\ 1, & x \in \mathbb{R}^{n} \backslash B_{R+1},\end{cases}
$$


from (2.29) and (2.30) we infer on an application of Beppo Levi's theorem that

$$
\frac{1}{2} \int_{\mathbb{R}^{n}} \zeta^{2}|\nabla v(\cdot, s)|^{2}+\frac{1}{2} \int_{s_{2}}^{2} \int_{\mathbb{R}^{n}} \zeta^{2} v|\Delta v|^{2} \leq \frac{1}{2} \int_{\mathbb{R}^{n}} \zeta^{2}\left|\nabla v\left(\cdot, s_{2}\right)\right|^{2}+2 \int_{s_{2}}^{s} \int_{B_{R+1} \backslash B_{R}} v|\nabla v|^{2}
$$

for all $s \in\left(s_{2}, T\right)$, which in view of the evident inequalities $\chi_{\mathbb{R}^{n} \backslash B_{R+1}} \leq \zeta \leq \chi_{\mathbb{R}^{n} \backslash B_{R}}$ immediately yields both the claimed regularity properties and (2.28).

We can now establish the desired continuity property.

Lemma 2.7. Suppose that for some $p \in(0,1)$, the positive function $v_{0}$ belongs to $L^{p}\left(\mathbb{R}^{n}\right) \cap C^{0}\left(\mathbb{R}^{n}\right)$ and satisfies

$$
v_{0}(x) \rightarrow 0 \quad \text { as }|x| \rightarrow \infty .
$$

Then the minimal solution $v$ of (2.1) has the property that $v(\cdot, s) \in W^{1,2}\left(\mathbb{R}^{n}\right)$ for all $s>0$, that $\nabla v \in L^{2}\left(\mathbb{R}^{n} \times(0, \infty)\right)$, and that

$$
(0, \infty) \ni s \mapsto \int_{\mathbb{R}^{n}}|\nabla v(\cdot, s)|^{2} \quad \text { is continuous. }
$$

Proof. As our assumptions necessarily require that $v_{0} \in L^{1}\left(\mathbb{R}^{n}\right)$, a first application of Lemma 2.5 shows that indeed $\nabla v \in L^{2}\left(\mathbb{R}^{n} \times(0, \infty)\right)$, which due to the fact that also $v_{0} \in L^{2}\left(\mathbb{R}^{n}\right)$ may be combined with Lemma 2.6 to imply that $v(\cdot, s)$ belongs to $W^{1,2}\left(\mathbb{R}^{n}\right)$ actually for all $s>0$. To verify the continuity property (2.32), we fix $s_{\star}>0$ and $\varepsilon>0$, and note that since $v_{0} \in L^{p}\left(\mathbb{R}^{n}\right)$, once more employing Lemma 2.5 we see that

$$
I:=\int_{0}^{\infty} \int_{\mathbb{R}^{n}} v^{p-1}|\nabla v|^{2}
$$

is finite. In particular, this entails that it is possible to find $s_{2}>0$ such that $s_{2} \in\left(s_{\star}-1, s_{\star}\right)$ and

$$
\int_{\mathbb{R}^{n}} v^{p-1}\left(\cdot, s_{2}\right)\left|\nabla v\left(\cdot, s_{2}\right)\right|^{2} \leq c_{1}:=\frac{I}{\min \left\{\frac{s_{\star}}{2}, 1\right\}}
$$

for otherwise we could draw the absurd conclusion that

$$
I \geq \int_{\max \left\{\frac{s_{\star}}{2}, s_{\star}-1\right\}}^{s_{\star}} \int_{\mathbb{R}^{n}} v^{p-1}|\nabla v|^{2}>c_{1} \cdot\left(s_{\star}-\max \left\{\frac{s_{\star}}{2}, s_{\star}-1\right\}\right)=c_{1} \cdot \min \left\{\frac{s_{\star}}{2}, 1\right\}=I .
$$

We next use that $p<1$ in choosing $\eta>0$ small enough such that

$$
c_{1} \eta^{1-p}<\frac{\varepsilon}{6} \quad \text { and } \quad 4 \eta^{2-p} I<\frac{\varepsilon}{6},
$$

and thereupon rely on the uniform spatial decay property of $v$, as asserted by Lemma 2.3, to find some suitably large $R>0$ fulfilling

$$
v(x, s)<\eta \quad \text { for all } x \in \mathbb{R}^{n} \backslash B_{R} \text { and all } s>0 .
$$

Finally, writing

$$
s_{\star \star}:=\max \left\{\frac{s_{\star}}{2}, s_{2}\right\} \in\left(s_{\star}-1, s_{\star}\right),
$$


we know from the fact that $v$ is a classical solution that $\nabla v$ is continuous, and hence uniformly continuous, in $\bar{B}_{R+1} \times\left[s_{\star \star}, s_{\star}+1\right]$, whence in particular we can pick $\delta \in\left(0, \min \left\{1, s_{\star}-s_{\star \star}\right\}\right)$ such that

$$
\left.\left|\int_{B_{R+1}}\right| \nabla v(\cdot, s)\right|^{2}-\int_{B_{R+1}}\left|\nabla v\left(\cdot, s_{\star}\right)\right|^{2} \mid<\frac{\varepsilon}{3} \quad \text { for all } s \in\left(s_{\star}-\delta, s_{\star}+\delta\right) \text {. }
$$

Now for $s>0$, we split

$$
\int_{\mathbb{R}^{n}}|\nabla v(\cdot, s)|^{2}-\int_{\mathbb{R}^{n}}\left|\nabla v\left(\cdot, s_{\star}\right)\right|^{2}=I_{1}(s)+I_{2}(s)+I_{3}
$$

where

$$
I_{1}(s):=\int_{B_{R+1}}|\nabla v(\cdot, s)|^{2}-\int_{B_{R+1}}\left|\nabla v\left(\cdot, s_{\star}\right)\right|^{2}
$$

as well as

$$
I_{2}(s):=\int_{\mathbb{R}^{n} \backslash B_{R+1}}|\nabla v(\cdot, s)|^{2} \quad \text { and } \quad I_{3}:=\int_{\mathbb{R}^{n} \backslash B_{R+1}}\left|\nabla v\left(\cdot, s_{\star}\right)\right|^{2},
$$

and where according to (2.37) we already know that

$$
\left|I_{1}(s)\right|<\frac{\varepsilon}{3} \quad \text { for all } s \in\left(s_{\star}-\delta, s_{\star}+\delta\right) .
$$

In order to estimate the two remaining terms on the right of (2.38), we first note that as a consequence of (2.35), (2.33) and the left inequality in (2.34),

$$
\begin{aligned}
\int_{\mathbb{R}^{n} \backslash B_{R}}\left|\nabla v\left(\cdot, s_{2}\right)\right|^{2} & =\int_{\mathbb{R}^{n} \backslash B_{R}} v^{1-p}\left(\cdot, s_{2}\right) \cdot v^{p-1}\left(\cdot, s_{2}\right)\left|\nabla v\left(\cdot, s_{2}\right)\right|^{2} \\
& \leq \eta^{1-p} \int_{\mathbb{R}^{n} \backslash B_{R}} v^{p-1}\left(\cdot, s_{2}\right)\left|\nabla v\left(\cdot, s_{2}\right)\right|^{2} \\
& \leq \eta^{1-p} c_{1} \\
& <\frac{\varepsilon}{6} .
\end{aligned}
$$

Therefore, Lemma 2.6 guarantees that

$$
\int_{\mathbb{R}^{n} \backslash B_{R+1}}|\nabla v(\cdot, s)|^{2}<\frac{\varepsilon}{6}+4 \int_{s_{2}}^{s} \int_{B_{R+1} \backslash B_{R}} v|\nabla v|^{2} \quad \text { for all } s>s_{2},
$$

where again by (2.35), in view of the definition of $I$, and by the second restriction in (2.34) we see that

$$
\begin{aligned}
4 \int_{s_{2}}^{s} \int_{B_{R+1} \backslash B_{R}} v|\nabla v|^{2} & \leq 4 \int_{0}^{\infty} \int_{\mathbb{R}^{n} \backslash B_{R}} v|\nabla v|^{2} \\
& \leq 4 \eta^{2-p} \int_{0}^{\infty} \int_{\mathbb{R}^{n} \backslash B_{R}} v^{p-1}|\nabla v|^{2} \\
& \leq 4 \eta^{2-p} I \\
& <\frac{\varepsilon}{6} \quad \text { for all } s>s_{2},
\end{aligned}
$$


so that in fact

$$
\int_{\mathbb{R}^{n} \backslash B_{R+1}}|\nabla v(\cdot, s)|^{2}<\frac{\varepsilon}{3} \quad \text { for all } s>s_{2} .
$$

Since according to our choice of $\delta$ and our definition (2.36) of $s_{\star \star}$ we know that

$$
s_{2}+\delta<s_{2}+\left(s_{\star}-s_{\star \star}\right) \leq s_{2}+\left(s_{\star}-s_{2}\right)=s_{\star},
$$

this firstly shows that

$$
\left|I_{2}(s)\right|<\frac{\varepsilon}{3} \quad \text { for all } s \in\left(s_{\star}-\delta, s_{\star}+\delta\right),
$$

and thus clearly warrants that also

$$
\left|I_{3}\right|=\left|I_{2}\left(s_{\star}\right)\right|<\frac{\varepsilon}{3} .
$$

In light of (2.38), in conjunction with (2.39) this shows that

$$
\left.\left|\int_{\mathbb{R}^{n}}\right| \nabla v(\cdot, s)\right|^{2}-\int_{\mathbb{R}^{n}}\left|\nabla v\left(\cdot, s_{\star}\right)\right|^{2} \mid<\varepsilon \quad \text { for all } s \in\left(s_{\star}-\delta, s_{\star}+\delta\right)
$$

and thereby completes the proof of (2.32).

\section{Classical unit-mass solutions of (1.2). Proof of Theorem 1.2}

Having derived some properties of solutions to (2.1), we proceed to use these for the construction of solutions to (1.2) through an appropriate transformation involving the functions introduced in the following.

Definition 3.1. Given a positive function $u_{0} \in C^{0}\left(\mathbb{R}^{n}\right) \cap L^{p}\left(\mathbb{R}^{n}\right)$ for some $p \in(0,1)$ which satisfies $\int_{\mathbb{R}^{n}} u_{0}=1$ an well as $u_{0}(x) \rightarrow 0$ as $|x| \rightarrow \infty$, we let $v$ be the minimal solution of (2.1) emanating from initial data $v_{0}:=u_{0}$ and set

$$
K(s):=\int_{\mathbb{R}^{n}}|\nabla v(\cdot, s)|^{2}, \quad s \in(0, \infty),
$$

and we let $H:[0, \infty) \rightarrow[0, \infty)$ by

$$
H(s):=s-\int_{0}^{s} \int_{0}^{\sigma} K(\tau) d \tau d \sigma, \quad s \in[0, \infty) .
$$

Moreover we define

$$
G(s):=\frac{1}{1-\int_{0}^{s} K(\tau) d \tau}, \quad s \in[0, \infty)
$$

as well as

$$
g(t):=G(h(t)), \quad t \in[0, \infty)
$$

and let

$$
u(x, t):=g(t) v(x, h(t)), \quad x \in \mathbb{R}^{n}, t \in[0, \infty),
$$

and

$$
L(t):=\int_{\mathbb{R}^{n}}|\nabla u(\cdot, t)|^{2}, \quad t \in(0, \infty) .
$$


In order to make sure that $H$ is surjective, let us recall the following from [7, Prop. 1.3].

Lemma 3.2. Let $v_{0} \in C^{0}\left(\mathbb{R}^{n}\right)$ positive and let $v$ be a global positive classical solution of (2.1). Then for any $R>0$ we have

$$
\inf _{x \in B_{R}}\{s v(x, s)\} \rightarrow \infty \quad \text { as } s \rightarrow \infty
$$

Indeed, this asserts invertibility of $H$, as contained in the next statement.

Lemma 3.3. Suppose that for some $p \in(0,1), u_{0} \in L^{p}\left(\mathbb{R}^{n}\right) \cap C^{0}\left(\mathbb{R}^{n}\right)$ is positive and satisfies $\int_{\mathbb{R}^{n}} u_{0}=1$ as well as $u_{0}(x) \rightarrow 0$ as $|x| \rightarrow \infty$. Then the function $H:[0, \infty) \rightarrow[0, \infty)$ defined in (3.2) is bijective and

$$
h:=H^{-1}:[0, \infty) \rightarrow[0, \infty)
$$

is well-defined. Moreover, $h$ belongs to $C^{2}((0, \infty)) \cap C^{0}([0, \infty))$, and for $t \in(0, \infty)$ we have

$$
h^{\prime}(t)=\frac{1}{H^{\prime}(h(t))} \quad \text { and } \quad h^{\prime \prime}(t)=-\frac{1}{\left.H^{\prime}(h(t))\right)^{2}} H^{\prime \prime}(h(t)) h^{\prime}(t)=-\frac{H^{\prime \prime}(h(t))}{\left.H^{\prime}(h(t))\right)^{3}},
$$

where $H^{\prime}(s)>0$ for all $s \in(0, \infty)$.

Proof. Due to the conditions on $u_{0}$, Lemma 2.7 asserts that $K$ is continuous on $(0, \infty)$ and Lemma 2.5 additionally shows that $K \in L^{1}((0, \infty))$ and correspondingly $H \in C^{2}((0, \infty)) \cap C^{0}([0, \infty))$. Furthermore, again according to Lemma 2.5

$$
H^{\prime}(s)=1-\int_{0}^{s} K(\tau) d \tau=\int_{\mathbb{R}^{n}} v(\cdot, s)>0 \quad \text { for all } s \in(0, \infty) .
$$

Hence, $H$ is strictly monotone and thus injective. Moreover, by Lemma 3.2 we can find $s_{0}>0$ such that for all $s>s_{0}$ we have $\inf _{x \in B_{1}} s v(x, s) \geq \frac{1}{\left|B_{1}\right|}$. With this, we see, again using Lemma 2.5, that

$$
\begin{aligned}
H(s) & =\int_{0}^{s}\left(\int_{\mathbb{R}^{n}} u_{0}-\int_{0}^{\sigma} K(\tau) d \tau\right) d \sigma=\int_{0}^{s} \int_{\mathbb{R}^{n}} v(s) d s \geq \int_{s_{0}}^{s}\left\{\int_{B_{1}} \sigma v(x, \sigma) d x\right\} \cdot \frac{1}{\sigma} d \sigma \\
& \geq \int_{s_{0}}^{s}\left|B_{1}\right| \inf _{x \in B_{1}}\{\sigma v(x, \sigma)\} \cdot \frac{1}{\sigma} d \sigma \geq \int_{s_{0}}^{s} \frac{1}{\sigma} d \sigma \rightarrow \infty
\end{aligned}
$$

as $s \rightarrow \infty$ and we can infer surjectivity of $H$. Altogether, we see that $h \in C^{0}([0, \infty))$ is well-defined. In addition, positivity of $H^{\prime}$ also shows that (3.8) holds and $h \in C^{2}((0, \infty))$.

Let us list some regularity properties and further connections between the functions introduced in Definition 3.1 and in (3.7).

Lemma 3.4. Let $u_{0} \in L^{p}\left(\mathbb{R}^{n}\right) \cap C^{0}\left(\mathbb{R}^{n}\right)$ for some $p \in(0,1)$ be positive with $\int_{\mathbb{R}^{n}} u_{0}=1$ Then $K \in$ $L^{1}((0, \infty)), G, g \in C^{1}((0, \infty)) \cap C^{0}([0, \infty)), L \in C^{0}((0, \infty)) \cap L_{l o c}^{1}([0, \infty)), u \in C^{2,1}\left(\mathbb{R}^{n} \times(0, \infty)\right) \cap$ 
$C^{0}\left(\mathbb{R}^{n} \times[0, \infty)\right)$ and

$$
\begin{aligned}
G(s) & =\frac{1}{H^{\prime}(s)} & & \text { for all } s>0, \\
h^{\prime}(t) & =g(t) & & \text { for all } t>0, \\
G^{\prime}(s) & =G^{2}(s) K(s) & & \text { for all } s>0, \\
L(t) & =G^{2}(h(t)) K(h(t)) & & \text { for all } t>0, \\
g^{\prime}(t) & =g(t) L(t) & & \text { for all } t>0 .
\end{aligned}
$$

Proof. Since $H^{\prime}(s)=1-\int_{0}^{s} K(\sigma) d \sigma$, obviously $G(s)=\frac{1}{H^{\prime}(s)}$ for $s>0$ and $G \in C^{1}((0, \infty))$ follows from Lemma 3.3. Lemma 2.5 warrants that $K \in L^{1}((0, \infty))$, admitting the conclusion that $G \in C^{0}([0, \infty))$. The asserted regularity of $g$ thereupon becomes an immediate consequence of (3.4). Recalling that $v \in C^{2,1}\left(\mathbb{R}^{n} \times(0, \infty)\right) \cap C^{0}\left(\mathbb{R}^{n} \times[0, \infty)\right)$ and $h \in C^{1}((0, \infty)) \cap C^{0}([0, \infty))$, we find that $u \in C^{2,1}\left(\mathbb{R}^{n} \times\right.$ $(0, \infty)) \cap C^{0}\left(\mathbb{R}^{n} \times[0, \infty)\right)$.

By (3.8), (3.10) and (3.4), $h^{\prime}(t)=\frac{1}{H^{\prime}(h(t))}=G(h(t))=g(t)$ for all $t \in(0, \infty)$. Straightforward application of the chain rule combined with (3.10) moreover shows that

$$
G^{\prime}(s)=-\frac{1}{\left(H^{\prime}(s)\right)^{2}} H^{\prime \prime}(s)=G^{2}(s) K(s), \quad s \in(0, \infty) .
$$

According to the definitions of $K, g$ and $u$, for any $t>0$ we have

$$
G^{2}(h(t)) K(h(t))=g^{2}(t) \int_{\mathbb{R}^{n}} \mid \nabla v\left(x,\left.h(t)\right|^{2} d x=\int_{\mathbb{R}^{n}}|\nabla(g(t) v(x, h(t)))|^{2} d x=\int_{\mathbb{R}^{n}}|\nabla u(x, t)|^{2} d x=L(t)\right.
$$

and from the known regularity properties of $G, K, h$ we can infer $L \in C^{0}((0, \infty)) \cap L_{l o c}^{1}([0, \infty))$. Differentiation of (3.4) together with (3.12), (3.13) leads to

$$
g^{\prime}(t)=G^{\prime}(h(t)) h^{\prime}(t)=G^{2}(h(t)) K(h(t)) h^{\prime}(t)=L(t) g(t), \quad t \in(0, \infty) .
$$

Lemma 3.5. Suppose that for some $p \in(0,1), u_{0} \in L^{p}\left(\mathbb{R}^{n}\right) \cap C^{0}\left(\mathbb{R}^{n}\right)$ is positive and satisfies $\int_{\mathbb{R}^{n}} u_{0}=1$ as well as $u_{0}(x) \rightarrow 0$ as $|x| \rightarrow \infty$. Let $v$ denote the minimal solution of (2.1) emanating from $v_{0}:=u_{0}$, and let $u$ be defined by (3.5) with $g$ and $h$ given by (3.4) and (3.7). Then $u$ is a positive classical solution of (1.2) in $\mathbb{R}^{n} \times(0, \infty)$ with the property that

$$
\int_{\mathbb{R}^{n}} u(\cdot, t)=1 \quad \text { for all } t>0
$$

Moreover, for all $T>0$ we have

$$
\int_{0}^{T} \int_{\mathbb{R}^{n}} u|\Delta u|^{2}<\infty
$$

and

$$
\int_{0}^{T} \int_{\mathbb{R}^{n}} u|\nabla u|^{2}<\infty .
$$


Proof. From Lemma 3.4 we know that the function $u$ satisfies $u \in C^{2,1}\left(\mathbb{R}^{n} \times(0, \infty)\right) \cap C^{0}\left(\mathbb{R}^{n} \times[0, \infty)\right)$, moreover (3.16) and (3.17) are valid according to (3.5), Lemma 2.6 and Lemma 2.5, the latter again being applicable since $u_{0} \in L^{2}\left(\mathbb{R}^{n}\right)$ due to our assumptions. As $g(0)=G(h(0))=G(0)=1$, we have

$$
u(x, 0)=g(0) v(x, h(0))=v(x, 0)=u_{0}(x),
$$

whereas by (2.1), (3.11), (3.14) and (3.5) we find that

$$
\begin{aligned}
u_{t}(x, t) & =g(t) v_{s}(x, h(t)) h^{\prime}(t)+g^{\prime}(t) v(x, h(t)) \\
& =g(t) v(x, h(t)) \Delta v(x, h(t)) g(t)+g(t) L(t) v(x, h(t)) \\
& =u(x, t) \Delta u(x, t)+u(x, t) L(t) \quad \text { for all } x \in \mathbb{R}^{n} \text { and } t>0
\end{aligned}
$$

and that thus (1.2) holds. To see that the total mass remains constant, again letting $s:=h(t)$ we note that $u(x, t)=G(s) v(x, s)$ for all $x \in \mathbb{R}^{n}$ and $t>0$ and hence, by Lemma 2.5, indeed

$$
\int_{\mathbb{R}^{n}} u(x, t)=G(s) \int_{\mathbb{R}^{n}} v(x, s)=\frac{1}{1-\int_{0}^{s} K(\sigma) d \sigma}\left[\int_{\mathbb{R}^{n}} u_{0}-\int_{0}^{s} K(\sigma) d \sigma\right]=1
$$

for all $t>0$.

Proof of Theorem 1.2. All parts of the statement are immediate from Lemma 3.5 .

\section{Decay of $\frac{\mathcal{E}(t)}{t}$ for arbitrary initial data}

In the preparatory Lemma 4.1 and Lemma 4.2, we shall refer to the definition of the functions $\zeta_{R} \in$ $W^{1, \infty}\left(\mathbb{R}^{n}\right)$ which for $R>0$ we introduce by letting

$$
\zeta_{R}(x):= \begin{cases}1 & \text { if }|x| \leq R \\ R+1-|x| & \text { if } R<|x|<R+1, \\ 0 & \text { if }|x| \geq R+1\end{cases}
$$

By means of a corresponding cut-off procedure we can firstly provide a clean argument for the following functional inequality for nonnegative (sub-)unit-mass functions enjoying a certain additional regularity property.

Lemma 4.1. Let $\varphi \in C^{2}\left(\mathbb{R}^{n}\right)$ be nonnegative and such that

$$
\int_{\mathbb{R}^{n}} \varphi \leq 1
$$

as well as

$$
\int_{\mathbb{R}^{n}} \varphi|\nabla \varphi|^{2}<\infty
$$

Then

$$
\left\{\int_{\mathbb{R}^{n}}|\nabla \varphi|^{2}\right\}^{2} \leq \int_{\mathbb{R}^{n}} \varphi|\Delta \varphi|^{2}
$$


Proof. We only need to consider the case when the expression on the right-hand side of (4.4) is finite, in which using the cut-off functions from (4.1) we may integrate by parts to find that

$$
\int_{\mathbb{R}^{n}} \zeta_{R}^{2}|\nabla \varphi|^{2}=-\int_{\mathbb{R}^{n}} \zeta_{R}^{2} \varphi \Delta \varphi-2 \int_{\mathbb{R}^{n}} \zeta_{R}\left(\nabla \zeta_{R} \cdot \nabla \varphi\right) \varphi \quad \text { for all } R>0,
$$

where by the Cauchy-Schwarz inequality and (4.2),

$$
\begin{aligned}
-\int_{\mathbb{R}^{n}} \zeta_{R}^{2} \varphi \Delta \varphi & \leq\left\{\int_{\mathbb{R}^{n}} \zeta_{R}^{2} \varphi|\Delta \varphi|^{2}\right\}^{\frac{1}{2}} \cdot\left\{\int_{\mathbb{R}^{n}} \zeta_{R}^{2} \varphi\right\}^{\frac{1}{2}} \\
& \leq\left\{\int_{\mathbb{R}^{n}} \varphi|\Delta \varphi|^{2}\right\}^{\frac{1}{2}} \quad \text { for all } R>0
\end{aligned}
$$

because $\zeta_{R}^{2} \leq 1$ on $\mathbb{R}^{n}$. By the same token and the observations that $\nabla \zeta_{R} \equiv 0$ in $B_{R} \cup\left(\mathbb{R}^{n} \backslash B_{R+1}\right)$ and $\left|\nabla \zeta_{R}\right| \leq 1$ in $B_{R+1} \backslash B_{R}$, again using he Cauchy-Schwarz inequality and (4.2) we see that moreover

$$
\begin{aligned}
\left|-2 \int_{\mathbb{R}^{n}} \zeta_{R}\left(\nabla \zeta_{R} \cdot \nabla \varphi\right) \varphi\right| & \leq 2 \int_{B_{R+1} \backslash B_{R}} \varphi|\nabla \varphi| \\
& \leq 2\left\{\int_{B_{R+1} \backslash B_{R}} \varphi|\nabla \varphi|^{2}\right\}^{\frac{1}{2}} \cdot\left\{\int_{B_{R+1} \backslash B_{R}} \varphi\right\}^{\frac{1}{2}} \\
& \leq 2\left\{\int_{B_{R+1} \backslash B_{R}} \varphi|\nabla \varphi|^{2}\right\}^{\frac{1}{2}} \quad \text { for all } R>0
\end{aligned}
$$

so that our assumption that $\varphi|\nabla \varphi|^{2}$ belong to $L^{1}\left(\mathbb{R}^{n}\right)$ warrants that

$$
-2 \int_{\mathbb{R}^{n}} \zeta_{R}\left(\nabla \zeta_{R} \cdot \nabla \varphi\right) \varphi \rightarrow 0 \quad \text { as } R \rightarrow \infty
$$

Since finally

$$
\int_{\mathbb{R}^{n}} \zeta_{R}^{2}|\nabla \varphi|^{2} \rightarrow \int_{\mathbb{R}^{n}}|\nabla \varphi|^{2} \quad \text { as } R \rightarrow \infty
$$

by e.g. Beppo Levi's theorem, we thus obtain from (4.5)-(4.7) that indeed (4.4) holds.

Lemma 4.2. Let $t_{0} \geq 0$ and $T>t_{0}$, and suppose that

$$
w \in C^{2,1}\left(\mathbb{R}^{n} \times\left[t_{0}, T\right]\right) \quad \text { with } \quad\left[t_{0}, T\right] \ni t \mapsto \int_{\mathbb{R}^{n}}|\nabla w(\cdot, t)|^{2} \in C^{0}\left(\left[t_{0}, T\right]\right)
$$

is a nonnegative classical solution of

$$
w_{t}=w \Delta w+w \int_{\mathbb{R}^{n}}|\nabla w|^{2}, \quad x \in \mathbb{R}^{n}, t \in\left[t_{0}, T\right]
$$

which is such that

$$
\int_{t_{0}}^{T} \int_{\mathbb{R}^{n}} w|\Delta w|^{2}<\infty
$$


and

$$
\int_{t_{0}}^{T} \int_{\mathbb{R}^{n}} w|\nabla w|^{2}<\infty
$$

as well as

$$
\int_{\mathbb{R}^{n}} w(\cdot, t) \leq 1 \quad \text { for all } t \in\left(t_{0}, T\right) .
$$

Then

$$
\int_{\mathbb{R}^{n}}|\nabla w(\cdot, T)|^{2} \leq \int_{\mathbb{R}^{n}}\left|\nabla w\left(\cdot, t_{0}\right)\right|^{2} .
$$

Proof. For $R>0$, we again take $\zeta_{R} \in W^{1, \infty}\left(\mathbb{R}^{n}\right)$ as defined in (4.1) and integrate by parts using (4.9) to compute

$$
\begin{aligned}
\frac{1}{2} \int_{\mathbb{R}^{n}} \zeta_{R}^{2}|\nabla w(\cdot, T)|^{2}-\frac{1}{2} \int_{\mathbb{R}^{n}} \zeta_{R}^{2}\left|\nabla w\left(\cdot, t_{0}\right)\right|^{2} \\
=\int_{t_{0}}^{T} \int_{\mathbb{R}^{n}} \zeta_{R}^{2} \nabla w \cdot \nabla w_{t} \\
=-\int_{t_{0}}^{T} \int_{\mathbb{R}^{n}} \nabla \cdot\left(\zeta_{R}^{2} \nabla w\right) w_{t} \\
=-\int_{t_{0}}^{T} \int_{\mathbb{R}^{n}} \zeta_{R}^{2} w|\Delta w|^{2}+\int_{t_{0}}^{T}\left\{\int_{\mathbb{R}^{n}} \zeta_{R}^{2} w \Delta w\right\} \cdot\left\{\int_{\mathbb{R}^{n}}|\nabla w|^{2}\right\} \\
\quad-2 \int_{t_{0}}^{T} \int_{\mathbb{R}^{n}} \zeta_{R}\left(\nabla \zeta_{R} \cdot \nabla w\right) w \Delta w-2 \int_{t_{0}}^{T}\left\{\int_{\mathbb{R}^{n}} \zeta_{R}\left(\nabla \zeta_{R} \cdot \nabla w\right) w\right\} \cdot\left\{\int_{\mathbb{R}^{n}}|\nabla w|^{2}\right\} .
\end{aligned}
$$

Here by the Cauchy-Schwarz inequality and (4.12), for all $t \in\left(t_{0}, T\right)$ we estimate, again using that $\zeta_{R}^{2} \leq 1$,

$$
\begin{aligned}
\int_{\mathbb{R}^{n}} \zeta_{R}^{2} w \Delta w & \leq\left\{\int_{\mathbb{R}^{n}} \zeta_{R}^{2} w|\Delta w|^{2}\right\}^{\frac{1}{2}} \cdot\left\{\int_{\mathbb{R}^{n}} \zeta_{R}^{2} w\right\}^{\frac{1}{2}} \\
& \leq\left\{\int_{\mathbb{R}^{n}} w|\Delta w|^{2}\right\}^{\frac{1}{2}} \quad \text { for all } R>0
\end{aligned}
$$

so that since Lemma 4.1 in conjunction with (4.11) guarantees that

$$
\int_{\mathbb{R}^{n}}|\nabla w|^{2} \leq\left\{\int_{\mathbb{R}^{n}} w|\Delta w|^{2}\right\}^{\frac{1}{2}} \quad \text { for a.e. } t \in\left(t_{0}, T\right),
$$

it follows that

$$
\int_{t_{0}}^{T}\left\{\int_{\mathbb{R}^{n}} \zeta_{R}^{2} w \Delta w\right\} \cdot\left\{\int_{\mathbb{R}^{n}}|\nabla w|^{2}\right\} \leq \int_{t_{0}}^{T} \int_{\mathbb{R}^{n}} w|\Delta w|^{2} \quad \text { for all } R>0 .
$$


Next, once more since $\operatorname{supp} \nabla \zeta_{R} \subset B_{R+1} \backslash B_{R}$ and $\left|\nabla \zeta_{R}\right| \leq 1$ a.e. in $\mathbb{R}^{n}$, by means of the CauchySchwarz inequality we find that

$$
\begin{aligned}
-2 \int_{t_{0}}^{T} \int_{\mathbb{R}^{n}} \zeta_{R}^{2}\left(\nabla \zeta_{R} \cdot \nabla w\right) w \Delta w & \leq 2 \int_{t_{0}}^{T} \int_{B_{R+1} \backslash B_{R}} w|\nabla w| \cdot|\Delta w| \\
& \leq 2\left\{\int_{t_{0}}^{T} \int_{B_{R+1} \backslash B_{R}} w|\Delta w|^{2}\right\}^{\frac{1}{2}} \cdot\left\{\int_{t_{0}}^{T} \int_{B_{R+1} \backslash B_{R}} w|\nabla w|^{2}\right\}^{\frac{1}{2}}
\end{aligned}
$$

and that, again thanks to (4.12),

$$
\begin{aligned}
-2 \int_{t_{0}}^{T}\left\{\int_{\mathbb{R}^{n}} \zeta_{R}\left(\nabla \zeta_{R} \cdot \nabla w\right) w\right\} \cdot\left\{\int_{\mathbb{R}^{n}}|\nabla w|^{2}\right\} & \leq 2 \int_{t_{0}}^{T}\left\{\int_{B_{R+1} \backslash B_{R}} w|\nabla w|\right\} \cdot\left\{\int_{\mathbb{R}^{n}}|\nabla w|^{2}\right\} \\
& \leq 2 c_{1} \int_{t_{0}}^{T} \int_{B_{R+1} \backslash B_{R}} w|\nabla w| \\
& \leq 2 c_{1}\left\{\int_{t_{0}}^{T} \int_{B_{R+1} \backslash B_{R}} w|\nabla w|^{2}\right\}^{\frac{1}{2}} \cdot\left\{\int_{t_{0}}^{T} \int_{B_{R+1} \backslash B_{R}} w\right\}^{\frac{1}{2}} \\
& \leq 2 c_{1} \sqrt{T}\left\{\int_{t_{0}}^{T} \int_{B_{R+1} \backslash B_{R}} w|\nabla w|^{2}\right\}^{\frac{1}{2}}
\end{aligned}
$$

for all $R>0$, where $c_{1}:=\|\nabla w\|_{L^{\infty}\left(\left(t_{0}, T\right) ; L^{2}(\Omega)\right)}$ is finite according to the second regularity requirement contained in (4.8). In summary, from (4.14)-(4.17) we infer that

$$
\begin{aligned}
\frac{1}{2} \int_{\mathbb{R}^{n}} \zeta_{R}^{2}|\nabla w(\cdot, T)|^{2}+\int_{t_{0}}^{T} \int_{\mathbb{R}^{n}} \zeta_{R}^{2} w|\Delta w|^{2} \leq & \frac{1}{2} \int_{\mathbb{R}^{n}} \zeta_{R}^{2}\left|\nabla w\left(\cdot, t_{0}\right)\right|^{2} \\
& +\int_{t_{0}}^{T} \int_{\mathbb{R}^{n}} w|\Delta w|^{2} \\
& +2\left\{\int_{t_{0}}^{T} \int_{B_{R+1} \backslash B_{R}} w|\Delta w|^{2}\right\}^{\frac{1}{2}} \cdot\left\{\int_{t_{0}}^{T} \int_{B_{R+1} \backslash B_{R}} w|\nabla w|^{2}\right\}^{\frac{1}{2}} \\
& +2 c_{1} \sqrt{T}\left\{\int_{t_{0}}^{T} \int_{B_{R+1} \backslash B_{R}} w|\nabla w|^{2}\right\}^{\frac{1}{2}} \text { for all } R>0 .
\end{aligned}
$$

Now since Beppo Levi's theorem ensures that as $R \rightarrow \infty$ we have

$$
\int_{\mathbb{R}^{n}} \zeta_{R}^{2}|\nabla w(\cdot, t)|^{2} \rightarrow \int_{\mathbb{R}^{n}}|\nabla w(\cdot, t)|^{2} \quad \text { for all } t \in\left[t_{0}, T\right]
$$

and

$$
\int_{t_{0}}^{T} \int_{\mathbb{R}^{n}} \zeta_{R}^{2} w|\Delta w|^{2} \rightarrow \int_{t_{0}}^{T} \int_{\mathbb{R}^{n}} w|\Delta w|^{2}
$$

and since the inclusions $w|\Delta w|^{2} \in L^{1}\left(\mathbb{R}^{n} \times\left(t_{0}, T\right)\right)$ and $w|\nabla w|^{2} \in L^{1}\left(\mathbb{R}^{n} \times\left(t_{0}, T\right)\right)$ asserted by (4.10) and (4.11) entail that

$$
\int_{t_{0}}^{T} \int_{B_{R+1} \backslash B_{R}} w|\Delta w|^{2} \rightarrow 0 \quad \text { and } \quad \int_{t_{0}}^{T} \int_{B_{R+1} \backslash B_{R}} w|\nabla w|^{2} \rightarrow 0 \quad \text { as } R \rightarrow \infty,
$$


on taking $R \rightarrow \infty$ in (4.18) we conclude that

$$
\frac{1}{2} \int_{\mathbb{R}^{n}}|\nabla w(\cdot, T)|^{2}+\int_{t_{0}}^{T} \int_{\mathbb{R}^{n}} w|\Delta w|^{2} \leq \frac{1}{2} \int_{\mathbb{R}^{n}}\left|\nabla w\left(\cdot, t_{0}\right)\right|^{2}+\int_{t_{0}}^{T} \int_{\mathbb{R}^{n}} w|\Delta w|^{2},
$$

and that hence (4.13) is valid.

Lemma 4.3. Let $R>0$. The the solution $\varphi_{R} \in C^{2}\left(\bar{B}_{R}\right)$ of $-\Delta \varphi_{R}=1$ in $B_{R}$ with $\varphi_{R}=0$ on $\partial B_{R}$ is given by

$$
\varphi_{R}(x):=\frac{R^{2}-|x|^{2}}{2 n}, \quad x \in \bar{B}_{R}
$$

and hence for each $R>0$ we have

$$
\varphi_{R}(x)=R^{2} \varphi_{1}\left(\frac{x}{R}\right) \quad \text { for all } x \in B_{R} \quad \text { and } \quad\left\|\varphi_{R}\right\|_{L^{1}\left(B_{R}\right)}=\frac{2 \omega_{n}}{n^{2}(n+1)} R^{n+2},
$$

where $\omega_{n}:=\left|\partial B_{1}\right|$.

Proof of Proposition 1.3. Since for all $t_{0}>0$ and $T>0$, Theorem 1.2 asserts that $u \in C^{2,1}\left(\mathbb{R}^{n} \times\right.$ $\left.\left[t_{0}, T\right]\right)$ and that $\left[t_{0}, T\right] \ni t \mapsto \int_{\mathbb{R}^{n}}|\nabla u(\cdot, t)|^{2}$ is continuous, whereas Lemma 3.5 says that $u|\Delta u|^{2}$ and $u|\nabla u|^{2}$ belong to $L^{1}\left(\mathbb{R}^{n} \times\left(t_{0}, T\right)\right)$, the claimed monotonicity property is a consequence of Lemma 4.2 , If (1.12) was false, we could thus find $c_{1}>0$ such that $\int_{\mathbb{R}^{n}}|\nabla u(\cdot, t)|^{2} \geq c_{1}$ for all $t>0$, which in view of (1.2) would imply that

$$
u_{t} \geq u \Delta u+c_{1} u \quad \text { for all } x \in \mathbb{R}^{n} \text { and } t>0 .
$$

In order to argue from this to a contradiction to the fact that $(0, \infty) \ni t \mapsto \int_{\mathbb{R}^{n}} u(\cdot, t)$ is bounded, let us fix some conveniently large $R>0$ such that for the function $\varphi_{R}$ from Lemma 4.3 we have

$$
\frac{c_{1}}{2} \int_{B_{R}} \varphi_{R}>1
$$

Then since $u_{0}$ was assumed to be positive throughout $\mathbb{R}^{n}$, it is possible to find some suitably small $c_{2} \in\left(0, \frac{c_{1}}{2}\right)$ such that

$$
u_{0}(x)>c_{2} \varphi_{R}(x) \quad \text { for all } x \in \bar{B}_{R}
$$

whereupon we define

$$
\underline{u}(x, t):=y(t) \varphi_{R}(x), \quad x \in \bar{B}_{R}, t \geq 0
$$

with

$$
y(t):=c_{2} e^{\frac{c_{1}}{2} t}, \quad t \geq 0 .
$$

Then (4.20) warrants that $u(\cdot, 0)>\underline{u}(\cdot, 0)$ in $\bar{B}_{R}$, whereas clearly $u>\underline{u}=0$ on $\partial B_{R} \times[0, \infty)$. Since writing $t_{0}:=\frac{2}{c_{1}} \ln \frac{c_{1}}{2 c_{2}}>0$ we have

$$
y(t) \leq \frac{c_{1}}{2}=y\left(t_{0}\right) \quad \text { for all } t \in\left(0, t_{0}\right)
$$


it particularly follows from (4.21) that $y^{\prime}=\frac{c_{1}}{2} y \leq c_{1} y-y^{2}$ on $\left(0, t_{0}\right)$ and that hence

$$
\begin{aligned}
\underline{u}_{t}-\underline{u} \Delta \underline{u}-c_{1} \underline{u} & =y^{\prime} \varphi_{R}-y^{2} \varphi_{R} \Delta \varphi_{R}-c_{1} y \varphi_{R} \\
& =\left\{y^{\prime}+y^{2}-c_{1} y\right\} \cdot \varphi_{R} \\
& \leq 0 \quad \text { in } B_{R} \times\left(0, t_{0}\right),
\end{aligned}
$$

because $-\Delta \varphi_{R}=1$ in $B_{R}$. Consequently, a comparison principle $([24])$ ensures that $\underline{u} \leq u$ in $\bar{B}_{R} \times\left[0, t_{0}\right]$ and that thus, thanks to the latter relation in (4.22) and (4.19),

$$
\int_{\mathbb{R}^{n}} u\left(\cdot, t_{0}\right) \geq \int_{B_{R}} \underline{u}\left(\cdot, t_{0}\right)=y\left(t_{0}\right) \int_{B_{R}} \varphi_{R}>1 .
$$

This is incompatible with the identity $\int_{\mathbb{R}^{n}} u\left(\cdot, t_{0}\right)=1$ obtained in Theorem 1.2 and thereby establishes (1.12).

\section{$5 \quad$ Logarithmic growth of $\mathcal{E}$ for algebraically decaying data}

In the case when $u_{0}$ satisfies algebraic decay conditions, thanks to our rather precise knowledge on the transformation functions appearing in Definition 3.1 and Lemma 3.3 we can obtain the asymptotic properties of $\mathcal{E}$ claimed in Theorem 1.4 in quite a straightforward manner from the following known result on large time behavior in (2.1).

Lemma 5.1. Let $v_{0} \in C^{0}\left(\mathbb{R}^{n}\right) \cap L^{\infty}\left(\mathbb{R}^{n}\right)$ be positive, and let $v$ denote the solution of (2.1) from Lemma 2.1.

i) If there exist $\gamma>n$ and $C_{0}>0$ fulfilling

$$
v_{0}(x) \geq C_{0} \cdot(1+|x|)^{-\gamma} \quad \text { for all } x \in \mathbb{R}^{n},
$$

then

$$
\|v(\cdot, t)\|_{L^{1}\left(\mathbb{R}^{n}\right)} \geq c t^{-(\gamma-n) /(\gamma+2)} \quad \text { for all } t>1
$$

with some $c>0$.

ii) If $v_{0} \in L^{p}\left(\mathbb{R}^{n}\right)$ for some $p \in(0,1)$, then one can find $C>0$ such that

$$
\|v(\cdot, t)\|_{L^{1}\left(\mathbb{R}^{n}\right)} \leq C t^{-(1-p) /\left(1+\frac{2 p}{n}\right)} \quad \text { for all } t>0 .
$$

Proof. For i), see [7, Theorem 1.6 (i)]; for ii), we refer to [7, Theorem 1.2].

Indeed, this quite directly entails the claimed properties of $\mathcal{E}$ when $u_{0}$ decays as indicated in Theorem 1.4 .

ProOF of Theorem 1.4 i) Letting $v$ denote the solution of (2.1) from Lemma 2.1 emanating from $v_{0}:=u_{0}$, from Lemma 5.1 we obtain $c_{1}>0$ such that $\|v(\cdot, s)\|_{L^{1}\left(\mathbb{R}^{n}\right)} \geq c_{1} s^{-\alpha}$ for all $s>1$, where $\alpha:=\frac{\gamma-n}{\gamma+2}$, so that by Theorem 1.2, (3.5) and (3.11) we have

$$
1=\|u(\cdot, t)\|_{L^{1}\left(\mathbb{R}^{n}\right)}=g(t)\|v(\cdot, h(t))\|_{L^{1}\left(\mathbb{R}^{n}\right)} \geq c_{1} g(t) h^{-\alpha}(t)=c_{1} h^{\prime}(t) h^{-\alpha}(t)
$$


whenever $h(t)>1$, that is, whenever $t>H(1)=: t_{0}$. This shows that

$$
g(t)=h^{\prime}(t) \leq \frac{1}{c_{1}} h^{\alpha}(t) \quad \text { for all } t>t_{0}
$$

and that thus

$$
1 \geq c_{1} h^{\prime}(t) h^{-\alpha}(t)=\frac{c_{1}}{1-\alpha}\left(h^{1-\alpha}\right)^{\prime}(t) \quad \text { for all } t>t_{0},
$$

meaning that

$$
h^{1-\alpha}(t)-h^{1-\alpha}\left(t_{0}\right)=\int_{t_{0}}^{t}\left(h^{1-\alpha}\right)^{\prime}(\tau) d \tau \leq \frac{1-\alpha}{c_{1}}\left(t-t_{0}\right) \quad \text { for all } t>t_{0},
$$

that is,

$$
h(t) \leq\left(\frac{1-\alpha}{c_{1}} t+h^{1-\alpha}\left(t_{0}\right)\right)^{\frac{1}{1-\alpha}} \quad \text { for all } t>t_{0} .
$$

Accordingly, by (5.1) and the validity of $(a+b)^{\alpha} \leq 2^{\alpha}\left(a^{\alpha}+b^{\alpha}\right)$ for all $a, b, \alpha \geq 0$,

$$
g(t) \leq \frac{1}{c_{1}}\left(\frac{1-\alpha}{c_{1}} t+h^{1-\alpha}\left(t_{0}\right)\right)^{\frac{\alpha}{1-\alpha}} \leq \frac{2^{\alpha}(1-\alpha)^{\frac{\alpha}{1-\alpha}}}{c_{1}^{\frac{1}{1-\alpha}}} t^{\frac{\alpha}{1-\alpha}}+\frac{2^{\alpha}}{c_{1}} h^{\alpha}\left(t_{0}\right) \quad \text { for all } t>t_{0} .
$$

As $g(t)=e^{\int_{0}^{t} L(\tau) d \tau}$ for all $t>0$ thanks to (3.14) and the fact that $g(0)=1$, (5.2) turns into the inequality

$$
\mathcal{E}(t)=\int_{0}^{t} L(\tau) d \tau=\ln (g(t)) \leq \ln \left(\frac{2^{\alpha}(1-\alpha)^{\frac{\alpha}{1-\alpha}}}{c_{1}^{\frac{1}{1-\alpha}}} t^{\frac{\alpha}{1-\alpha}}+\frac{2^{\alpha}}{c_{1}} h^{\alpha}\left(t_{0}\right)\right) \quad \text { for all } t>t_{0} .
$$

Now since for $t>\max \left\{t_{0}, \frac{c_{1}}{1-\alpha} h^{1-\alpha}\left(t_{0}\right)\right\}$ we have that $\frac{2^{\alpha}(1-\alpha)^{\frac{\alpha}{1-\alpha}}}{c_{1}^{\frac{1}{1-\alpha}}} t^{\frac{\alpha}{1-\alpha}}>\frac{2^{\alpha}}{c_{1}} h^{\alpha}\left(t_{0}\right)$, in view of the observation that

$$
\frac{\alpha}{1-\alpha}=\frac{1}{\frac{1}{\alpha}-1}=\frac{1}{\frac{\gamma+2}{\gamma-n}-1}=\frac{\gamma-n}{\gamma+2-(\gamma-n)}=\frac{\gamma-n}{n+2}
$$

we obtain (1.14) with $C:=\ln \left(2^{\alpha+1}(1-\alpha)^{\frac{\alpha}{1-\alpha}} c_{1}^{-\frac{1}{1-\alpha}}\right)$ and $T:=\max \left\{t_{0}, \frac{c_{1}}{1-\alpha} h^{1-\alpha}\left(t_{0}\right)\right\}$.

ii) Given $\varepsilon>0$, we define $p:=\frac{n}{\gamma-\varepsilon}$ and observe that then (1.15) shows that $u_{0} \in L^{p}\left(\mathbb{R}^{n}\right)$. Now proceeding as in part i), we let $v$ be the solution of (2.1) from Lemma 2.1 with $v_{0}:=u_{0}$, and first invoke Lemma 5.1 to obtain $c_{2}>0$ such that

$$
1=\|u(\cdot, t)\|_{L^{1}\left(\mathbb{R}^{n}\right)}=g(t)\|v(\cdot, h(t))\|_{L^{1}\left(\mathbb{R}^{n}\right)} \leq c_{2} g(t) h^{-\alpha}(t)=c_{2} h^{\prime}(t) h^{-\alpha}(t) \quad \text { for all } t>0,
$$

this time with $\alpha=\frac{1-p}{1+\frac{2 p}{n}}$, so that $g(t) \geq \frac{1}{c_{2}} h^{\alpha}(t)$ and $\left(h^{1-\alpha}\right)^{\prime}(t) \geq \frac{1-\alpha}{c_{2}}$ for all $t>0$. Consequently,

$$
h^{1-\alpha}(t)=\int_{0}^{t}\left(h^{1-\alpha}\right)^{\prime}(\tau) d \tau \geq \frac{1-\alpha}{c_{2}} t, \quad \text { for all } t>0
$$


and

$$
g(t) \geq \frac{1}{c_{2}} h^{\alpha}(t) \geq c_{2}^{-\frac{1}{1-\alpha}}(1-\alpha)^{\frac{\alpha}{1-\alpha}} t^{\frac{\alpha}{1-\alpha}} \quad \text { for all } t>0 .
$$

Since

$$
\frac{\alpha}{1-\alpha}=\frac{1}{\frac{1}{\alpha}-1}=\frac{1}{\frac{1+\frac{2 p}{n}}{1-p}-1}=\frac{1-p}{\frac{2 p}{n}+p}=\frac{1-p}{p} \cdot \frac{n}{2+n},
$$

from the identity $\mathcal{E}(t)=\ln (g(t)), t>0$, we obtain that

$$
\mathcal{E}(t) \geq \frac{1-p}{p} \cdot \frac{n}{2+n} \ln (t)-c_{3} \quad \text { for any } t>0
$$

with $c_{3}:=\frac{1}{1-\alpha}\left(\ln \left(c_{2}\right)-\alpha \ln (1-\alpha)\right)$. Since herein we have

$$
\frac{(1-p) n}{p}=\frac{\left(1-\frac{n}{\gamma-\varepsilon}\right) n}{\frac{n}{\gamma-\varepsilon}}=(\gamma-\varepsilon)\left(1-\frac{n}{\gamma-\varepsilon}\right)=\gamma-n-\varepsilon
$$

according to our definition of $p$, this yields the claimed inequality.

\section{$6 \quad$ Weakly sublinear growth of $\mathcal{E}$ for rapidly decreasing initial data}

\subsection{A lower estimate for $\mathcal{E}$ in terms of $\mathcal{L}$}

We next intend to examine how far imposing faster decay conditions on the initial data in (1.2) can enforce asymptotics of $\mathcal{E}$ different from that observed before for algebraically decreasing data. Our first step in this direction yields a lower estimate for $\mathcal{E}$ under a given hypothesis on the temporal decay of the solution to (2.1), formulated in terms of a rather general function $\ell$ fulfilling appropriately mild conditions.

Lemma 6.1. Assume that $\ell \in C^{0}([0, \infty))$ satisfies (1.17), (1.18) and (1.19). Let $u_{0} \in C^{0}\left(\mathbb{R}^{n}\right)$ be a positive function satisfying (1.6)-(1.8), and let $u$ and $v$ be as in Lemma 3.5. Suppose that there exist $C_{0}>0$ and $s_{0}>0$ such that

$$
\|v(\cdot, s)\|_{L^{1}\left(\mathbb{R}^{n}\right)} \leq C_{0} s^{-1} \cdot \ell^{-\frac{n+2}{n}}\left(\frac{1}{s}\right) \quad \text { for all } s>s_{0} .
$$

Then we can find $t_{0}>0$ and $C_{1}>0, C_{2} \geq 0$ such that with $\mathcal{L}$ as defined in (1.20) we have

$$
\mathcal{E}(t) \geq \ln \left(\left(\mathcal{L}^{-1}\right)^{\prime}\left(C_{1} t\right)\right)-C_{2} \quad \text { for all } t>t_{0} .
$$

Proof. From (6.3) we obtain that due to Theorem 1.2 and (3.5),

$$
1=g(t)\|v(\cdot, h(t))\|_{L^{1}} \leq C_{0} g(t) \frac{1}{h(t)} \ell^{-\frac{n+2}{n}}\left(\frac{1}{h(t)}\right) \quad \text { for } t>t_{1}:=\max \left\{H\left(s_{0}\right), H(1)\right\},
$$

which by (1.20) implies that

$$
g(t) \geq \frac{1}{C_{0}} \cdot h(t) \ell^{\frac{n+2}{n}}\left(\frac{1}{h(t)}\right)=\frac{1}{C_{0} \mathcal{L}^{\prime}(h(t))} \quad \text { for } t>t_{1}
$$


as well as, by (3.11),

$$
(\mathcal{L} \circ h)^{\prime}(t) \geq \frac{1}{C_{0}} \quad \text { for } t>t_{1}
$$

Taking into account that our choice of $t_{1}$ ensures that $h\left(t_{1}\right) \geq 1$ and that thus $\mathcal{L}\left(h\left(t_{1}\right)\right) \geq \mathcal{L}(1)=0$, we have

$$
h(t) \geq \mathcal{L}^{-1}\left(\mathcal{L}\left(h\left(t_{1}\right)\right)+\frac{t-t_{1}}{C_{0}}\right) \geq \mathcal{L}^{-1}\left(\frac{t-t_{1}}{C_{0}}\right) \quad \text { for all } t>t_{1}
$$

According to (1.18),$\frac{1}{\mathcal{L}^{\prime}(\cdot)}$ is monotone on $\left(\xi_{0}, \infty\right)$ and hence (6.5) implies that

$$
\mathcal{E}(t)=\ln (g(t)) \geq \ln \left(\frac{1}{C_{0} \mathcal{L}^{\prime}\left(\mathcal{L}^{-1}\left(c_{1}\left(t-t_{1}\right)\right)\right)}\right)=\ln \left(\left(\mathcal{L}^{-1}\right)^{\prime}\left(c_{1} t-c_{2}\right)\right)-\ln C_{0} \quad \text { for all } t>t_{2},
$$

where $c_{1}:=\frac{1}{C_{0}}, c_{2}:=c_{1} t_{1}$ and $t_{2}:=\max \left\{t_{1}, \frac{1}{c_{1}}\left(\mathcal{L}\left(\xi_{0}\right)+c_{2}\right)\right\}$. Once more employing monotonicity of $\left(\mathcal{L}^{-1}\right)^{\prime}$, we obtain (6.4) with $C_{1}:=\frac{c_{1}}{2}, C_{2}=\max \left\{0, \ln C_{0}\right\}$ and $t_{0}:=\max \left\{\frac{2 c_{2}}{c_{1}}, 2 c_{0} \mathcal{L}\left(\xi_{0}\right), t_{2}\right\}$.

We proceed to ensure (6.3) under appropriate conditions, relying on two statements on decay of solutions to (2.1) in $L^{q}\left(\mathbb{R}^{n}\right)$ for small $q>0$ and in $L^{\infty}\left(\mathbb{R}^{n}\right)$, respectively, as derived in [8].

Lemma 6.2. Suppose that $\ell \in C^{0}([0, \infty))$ satisfies (1.17) and is such that with some $\xi_{2}>0, a>0$ and $\lambda_{0}>0$ we have $\ell \in C^{2}\left(\left(0, \xi_{2}\right)\right)$ and that (1.22) and (1.23) are satisfied. Moreover, assume that $v_{0} \in C^{0}\left(\mathbb{R}^{n}\right)$ is positive, radially symmetric and nonincreasing with respect to $|x|$. Then if there exists $q_{0}>0$ such that

$$
v_{0}<\min \left\{\xi_{2}^{2}, \xi_{2}^{\frac{2}{1+q_{0}}}\right\} \quad \text { in } \mathbb{R}^{n}
$$

and if furthermore

$$
\int_{\mathbb{R}^{n}} \ell\left(v_{0}\right)<\infty
$$

one can find $s_{0}>0$ and $C>0$ such that the minimal solution $v$ of (2.1) satisfies

$$
\|v(\cdot, s)\|_{L^{1}\left(\mathbb{R}^{n}\right)} \leq C s^{-1} \ell^{-\frac{n+2}{n}}\left(\frac{1}{s}\right) \quad \text { for all } s>s_{0} .
$$

Proof. From [8, Lemma 3.6] and Fatou's lemma we infer that (6.6) and (6.7) ensure the existence of some $q \in\left(0, \min \left\{q_{0}, 1\right\}\right)$ as well as positive constants $c_{1}$ and $s_{1}$ such that

$$
\|v(\cdot, s)\|_{L^{q}\left(\mathbb{R}^{n}\right)} \leq c_{1} s^{-1} \ell^{-\frac{n+2 q}{n q}}\left(\frac{1}{s}\right) \quad \text { for all } s \geq s_{1} .
$$

Moreover, the additional assumption on radial symmetry and monotonicity of $v_{0}$ enables us to invoke [8, Theorem 1.3] which yields $c_{2}>0$ and $s_{2}>0$ fulfilling

$$
\|v(\cdot, s)\|_{L^{\infty}\left(\mathbb{R}^{n}\right)} \leq c_{2} s^{-1} \ell^{-\frac{2}{n}}\left(\frac{1}{s}\right) \quad \text { for all } s \geq s_{2} .
$$


Combining (6.8) with (6.9) we thus obtain

$$
\begin{aligned}
\int_{\mathbb{R}^{n}} v(\cdot, s) & =\int_{\mathbb{R}^{n}} v^{1-q}(\cdot, s) v^{q}(\cdot, s) \\
& \leq\|v(\cdot, s)\|_{L^{\infty}\left(\mathbb{R}^{n}\right)}^{1-q}\|v(\cdot, s)\|_{L^{q}\left(\mathbb{R}^{n}\right)}^{q} \\
& \leq c_{1}^{1-q} s^{-(1-q)} \ell^{-\frac{2}{n}(1-q)}\left(\frac{1}{s}\right) \cdot c_{2}^{q} s^{-q} \ell^{-\frac{n+2 q}{n q} q}\left(\frac{1}{s}\right) \\
& =c_{1}^{1-q} c_{2}^{q} s^{-1} \ell^{-\frac{n+2}{n}}\left(\frac{1}{s}\right)
\end{aligned}
$$

for all $s>\max \left\{s_{1}, s_{2}\right\}$.

\subsection{An upper estimate for $\mathcal{E}$ in terms of $\mathcal{L}$}

The following conditional information on $\mathcal{E}$ can be viewed as a counterpart of Lemma 6.1.

Lemma 6.3. Let $\ell \in C^{0}([0, \infty))$ satisfy (1.17), (1.18) and (1.19), let $u_{0} \in C^{0}\left(\mathbb{R}^{n}\right)$ be a positive function satisfying (1.6)-(1.8), and let $u$ and $v$ be as introduced in Lemma 3.5. Then if there exist $C_{0}>0$ and $t_{0}>0$ such that

$$
\|v(\cdot, s)\|_{L^{1}\left(\mathbb{R}^{n}\right)} \geq C_{0} s^{-1} \ell^{-\frac{n+2}{n}}\left(\frac{1}{s}\right) \quad \text { for all } s>s_{0} .
$$

we can find $t_{1}>0$ and $C_{1}>0, C_{2} \geq 0$ with the property that for the function $\mathcal{E}$ from (1.10) we have

$$
\mathcal{E}(t) \leq \ln \left(\left(\mathcal{L}^{-1}\right)^{\prime}\left(C_{1} t\right)\right)+C_{2} \quad \text { for any } t>t_{0} .
$$

Proof. Proceeding similarly to the proof of Lemma 6.1, we use (6.10), Theorem 1.2, (3.5), (1.20) and (3.11) to obtain

$$
g(t) \leq \frac{1}{C_{0} \mathcal{L}^{\prime}(h(t))} \quad \text { and } \quad(\mathcal{L} \circ h)^{\prime}(t) \leq \frac{1}{C_{0}} \quad \text { for } t>t_{1}:=H\left(s_{0}\right),
$$

so that

$$
\mathcal{L}(h(t)) \leq \mathcal{L}\left(h\left(t_{1}\right)\right)+\frac{t-t_{1}}{C_{0}} \leq c_{1} t \quad \text { for all } t>t_{2}:=\max \left\{t_{1}, C_{0} \mathcal{L}\left(h\left(t_{1}\right)\right)\right\}
$$

with $c_{1}:=\frac{2}{C_{0}}$, whence finally

$$
\mathcal{E}(t) \leq \ln \left(\left(\mathcal{L}^{-1}\right)^{\prime}\left(c_{1} t\right)\right), \quad \text { whenever } t>\max \left\{t_{2}, H\left(\xi_{0}\right)\right\}
$$

as desired, with $\xi_{0}$ as in (1.18).

In order to identify conditions ensuring (6.10), we make use of a comparison argument inspired by that in [8, Theorem 1.6] to establish a pointwise lower estimate for positive solutions of (2.1). 
Lemma 6.4. Let $\ell \in C^{0}\left([0, \infty)\right.$ be such that (1.17) holds, that $\ell$ is strictly increasing on $\left(0, \xi_{1}\right)$ for some $\xi_{1} \in(0, \infty]$, and that

$$
\frac{\xi \ell^{\prime}(\xi)}{\ell(\xi)} \rightarrow 0 \quad \text { as } \xi \searrow 0 .
$$

Moreover, assume that there exist $q \in(0,1)$ and $R_{\star}>\max \left\{\frac{1}{\sqrt[n]{\xi_{1}}}, \frac{1}{\sqrt[n]{\lim _{\xi} \xi_{1}} \ell(\xi)}\right\}$ such that the positive function $v_{0} \in C^{0}\left(\mathbb{R}^{n}\right) \cap L^{\infty}\left(\mathbb{R}^{n}\right)$ satisfies

$$
v_{0}(x) \geq\left\{\ell^{-1}\left(\frac{1}{|x|^{n}}\right)\right\}^{q} \quad \text { for all } x \in \mathbb{R}^{n} \backslash B_{R_{\star}} .
$$

Then with some $s_{0}>0$ and $C>0$, the corresponding minimal solution $v$ of (2.1) fulfills

$$
\|v(\cdot, s)\|_{L^{1}\left(\mathbb{R}^{n}\right)} \geq C s^{-1} \cdot \ell^{-\frac{n+2}{n}}\left(\frac{1}{s}\right) \quad \text { for all } s>s_{0} .
$$

Proof. We first observe that

$$
z(x, \sigma):=(s+1) v(x, s), \quad x \in \mathbb{R}^{n}, \sigma=\ln (s+1) \geq 0,
$$

defines a positive classical solution of

$$
\left\{\begin{array}{l}
z_{\sigma}=z \Delta z+z, \quad x \in \mathbb{R}^{n}, \sigma>0, \\
z(x, 0)=v_{0}(x), \quad x \in \mathbb{R}^{n},
\end{array}\right.
$$

and in order to estimate $z$ from below appropriately, given $\sigma>0$ we set

$$
R(\sigma):=\left\{\ell\left(e^{-\sigma}\right)\right\}^{-\frac{1}{n}}
$$

and then obtain from L'Hospital's rule that (6.12) entails that

$$
\lim _{\sigma \rightarrow \infty} \frac{2}{\sigma} \ln R(\sigma)=\lim _{\sigma \rightarrow \infty} \frac{-2}{n \sigma} \ln \left(\ell\left(e^{-\sigma}\right)\right)=\lim _{\sigma \rightarrow \infty} \frac{2}{n} \frac{\ell^{\prime}\left(e^{-\sigma}\right) e^{-\sigma}}{\ell\left(e^{-\sigma}\right)}=\lim _{\xi \searrow 0} \frac{\xi \ell^{\prime}(\xi)}{\ell(\xi)}=0 .
$$

Hence,

$$
\lim _{\sigma \rightarrow \infty}((q-1) \sigma+2 \ln R(\sigma))=\lim _{\sigma \rightarrow \infty}\left(-(1-q)+\frac{2}{\sigma} \ln R(\sigma)\right) \sigma=-\infty
$$

so that we can find $c_{1}>0$ with the property that

$$
(q-1) \sigma+2 \ln R(\sigma) \leq c_{1} \quad \text { for all } \sigma>0 .
$$

With $R_{\star}$ taken from (6.13), we now abbreviate

$$
\sigma_{\star}:=\frac{1}{q} \ln \frac{1}{\inf _{x \in B_{R_{\star}}} v_{0}(x)},
$$


and for $\sigma_{0}>\sigma_{\star}$ we let

$$
\delta\left(\sigma_{0}\right):=\frac{2 n e^{-q \sigma_{0}}}{R^{2}\left(\sigma_{0}\right)}
$$

and

$$
y(\sigma):=\left(\frac{e^{-\sigma}}{\delta\left(\sigma_{0}\right)}+1-e^{-\sigma}\right)^{-1}, \quad \sigma \geq 0,
$$

that is, we let $y$ be the solution of

$$
\left\{\begin{array}{l}
y^{\prime}(\sigma)=y(\sigma)-y^{2}(\sigma), \quad \sigma>0 \\
y(0)=\delta\left(\sigma_{0}\right)
\end{array}\right.
$$

Thus, if we define

$$
\underline{z}(x, \sigma):=y(\sigma) \varphi_{R\left(\sigma_{0}\right)}(x), \quad x \in B_{R\left(\sigma_{0}\right)}, \sigma \geq 0,
$$

with $\varphi_{R\left(\sigma_{0}\right)}$ as in Lemma 4.3, then $\underline{z}$ solves

$$
\begin{aligned}
\underline{z}_{\sigma}-\underline{z} \Delta \underline{z}-\underline{z} & =y^{\prime} \varphi_{R\left(\sigma_{0}\right)}-y^{2} \varphi_{R\left(\sigma_{0}\right)} \Delta \varphi_{R\left(\sigma_{0}\right)}-y \varphi_{R\left(\sigma_{0}\right)} \\
& =\left(y-y^{2}\right) \varphi_{R\left(\sigma_{0}\right)}+y^{2} \varphi_{R\left(\sigma_{0}\right)}-y \varphi_{R\left(\sigma_{0}\right)} \\
& =0, \quad x \in B_{R\left(\sigma_{0}\right)}, \sigma>0
\end{aligned}
$$

and evidently $\underline{z}(x, \sigma)=0 \leq z(x, \sigma)$ for all $x \in \partial B_{R\left(\sigma_{0}\right)}$ and $\sigma>0$. Moreover, using the montonicity of $\ell^{-1}$ in $\left[0, \frac{1}{R_{\star}^{n}}\right)$ we may employ (6.13) to see that for all $x \in B_{R\left(\sigma_{0}\right)} \backslash B_{R_{\star}}$ we can estimate

$$
u_{0}(x) \geq\left\{\ell^{-1}\left(\frac{1}{R^{n}\left(\sigma_{0}\right)}\right)\right\}^{q}=\left\{\ell^{-1}\left(\ell\left(e^{-\sigma_{0}}\right)\right)\right\}^{q}=e^{-q \sigma_{0}}
$$

so that since

$$
\varphi_{R\left(\sigma_{0}\right)}(x) \leq \frac{R^{2}\left(\sigma_{0}\right)}{2 n} \quad \text { in } B_{R\left(\sigma_{0}\right)},
$$

according to (6.18) for any such $x$ we have

$$
\frac{z(x, 0)}{\underline{z}(x, 0)}=\frac{v_{0}(x)}{\delta\left(\sigma_{0}\right) \varphi_{R\left(\sigma_{0}\right)}(x)} \geq \frac{e^{-q \sigma_{0}}}{\delta\left(\sigma_{0}\right) \cdot \frac{R^{2}\left(\sigma_{0}\right)}{2 n}}=1 .
$$

In the corresponding inner region, again by (6.19) we infer that

$$
\frac{z(x, 0)}{\underline{z}(x, 0)} \geq \frac{\inf _{x \in B_{R_{\star}}} v_{0}(x)}{\delta\left(\sigma_{0}\right) \cdot \frac{R^{2}\left(\sigma_{0}\right)}{2 n}}=e^{q \sigma_{0}} \cdot \inf _{x \in B_{R_{\star}}} v_{0}(x) \geq e^{q \sigma_{\star}} \cdot \inf _{x \in B_{R_{\star}}} v_{0}(x)=1 \quad \text { for all } x \in B_{R_{\star}}
$$

due to (6.17) and our restriction $\sigma_{0}>\sigma_{\star}$. A comparison argument ([24]) therefore shows that $z \geq \underline{z}$ in $B_{R\left(\sigma_{0}\right)} \times(0, \infty)$ and that thus, in particular,

$$
\begin{aligned}
\left\|z\left(\cdot, \sigma_{0}\right)\right\|_{L^{1}\left(\mathbb{R}^{n}\right)} & \geq\left\|\underline{z}\left(\cdot, \sigma_{0}\right)\right\|_{L^{1}\left(B_{R\left(\sigma_{0}\right)}\right)} \\
& =y\left(\sigma_{0}\right)\left\|\varphi_{R\left(\sigma_{0}\right)}\right\|_{L^{1}\left(B_{R\left(\sigma_{0}\right)}\right)} \\
& =\left(\frac{e^{-\sigma_{0}}}{\delta\left(\sigma_{0}\right)}+1-e^{-\sigma_{0}}\right)^{-1} \cdot \frac{2 \omega_{n}}{n^{2}(n+2)} R^{2+n}\left(\sigma_{0}\right)
\end{aligned}
$$


according to Lemma 4.3. As our choice of $\delta\left(\sigma_{0}\right)$ together with (6.16) ensures that herein

$$
\begin{aligned}
\frac{e^{-\sigma_{0}}}{\delta\left(\sigma_{0}\right)}+1-e^{-\sigma_{0}} & \leq \frac{e^{-\sigma_{0}}}{\delta\left(\sigma_{0}\right)}+1 \\
& =\frac{1}{2 n} e^{-(1-q) \sigma_{0}+2 \ln R\left(\sigma_{0}\right)}+1 \\
& \leq \frac{1}{2 n} e^{c_{1}}+1 \quad \text { for all } \sigma_{0}>\sigma_{\star},
\end{aligned}
$$

writing $c_{2}:=\left(\frac{1}{n} e^{2 c_{1}}+1\right)^{-1} \frac{2 \omega_{n}}{n^{2}(n+2)}$ we obtain

$$
\left\|z\left(\cdot, \sigma_{0}\right)\right\|_{L^{1}\left(\mathbb{R}^{n}\right)} \geq c_{2} R^{2+n}\left(\sigma_{0}\right) \quad \text { for all } \sigma_{0}>\sigma_{\star} .
$$

For arbitrary $s>e^{\sigma_{\star}}-1$, choosing $\sigma_{0}:=\ln (s+1)$ we see that thanks to the monotonicity of $\ell$ and (6.15) this implies that

$$
\begin{aligned}
\|v(\cdot, s)\|_{L^{1}\left(\mathbb{R}^{n}\right)} & \geq(s+1)^{-1}\|z(\cdot, \ln (s+1))\|_{L^{1}\left(\mathbb{R}^{n}\right)} \\
& \geq c_{2}(s+1)^{-1} \cdot \ell^{-\frac{n+2}{n}}\left(e^{-\ln (s+1)}\right) \\
& \geq c_{2}(s+1)^{-1} \cdot \ell^{-\frac{n+2}{n}}\left(\frac{1}{s+1}\right) \\
& \geq c_{2}(s+1)^{-1} \cdot \ell^{-\frac{n+2}{n}}\left(\frac{1}{s}\right)
\end{aligned}
$$

and thereby readily yields (6.14).

\subsection{Proof of Theorem 1.5}

We now only need to combine the results of the previous two sections to obtain our main result on asymptotic behavior of $\mathcal{E}$ for rapidly decreasing initial data.

Proof of Theorem 1.5. Part i) can be obtained by a straightforward combination of Lemma 6.3 with Lemma 6.4 and a comparison argument ([24]), whereas part ii) similarly results from Lemma 6.1 and Lemma 6.2.

\subsection{Examples}

\subsubsection{Exponentially decaying data. Proof of Corollary 1.6}

Proof of Corollary 1.6. Given $\kappa>0$ and $M \geq 2$ we let

$$
\ell(\xi)= \begin{cases}0, & \xi=0, \\ \ln ^{-\kappa}\left(\frac{M}{\xi}\right), & \xi \in\left(0, \frac{M}{2}\right), \\ \ln ^{-\kappa}(2), & \xi \geq \frac{M}{2} .\end{cases}
$$

This function obviously satisfies (1.17) and is continuous as well as strictly increasing on $\left(0, \xi_{1}\right)$ for $\xi_{1}=\frac{M}{2}$. That it moreover fulfills $(\underline{1.22})$ and (1.23) has been shown in [8, Lemma 3.9] for $\xi_{2}=\frac{M}{2}$, 
arbitrary $\lambda_{0}>0$ and $a=\kappa$ if $\kappa<1$ and $a=\frac{\left(1+\lambda_{0}\right)^{\kappa}-1}{\lambda_{0}}$ otherwise. This also entails (1.21), see [8, Lemma 2.1]. Finally, there exists $\xi_{0}>0$ such that $\xi_{0} \leq \xi \mapsto \ell^{\frac{n+2}{n}}\left(\frac{1}{\xi}\right)=\xi \ln ^{-\kappa \frac{n+2}{n}}(M \xi)$, i.e. (1.18).

Furthermore,

$$
\int_{1}^{\infty} \frac{1}{\xi} \ell^{-\frac{n+2}{n}}\left(\frac{1}{\xi}\right) d \xi=\int_{1}^{\infty} \frac{1}{\xi} \ln ^{\kappa \frac{n+2}{n}}(M \xi) d \xi=\infty
$$

and thus (1.19) holds.

The function $\ell^{-1}:\left[0, \ln ^{-\kappa} 2\right) \rightarrow\left[0, \frac{M}{2}\right)$ is given by $\ell^{-1}(\xi)=M e^{-\xi^{-\frac{1}{\kappa}}}$ for $\xi \in\left(0, \ln ^{-\kappa} 2\right)$. We compute $\mathcal{L}(t)=\int_{1}^{t} \frac{1}{\xi} \ell^{-\frac{n+2}{n}}\left(\frac{1}{\xi}\right) d \xi$ for $t>1$, so that we have

$$
\mathcal{L}(t)=\int_{1}^{t} \frac{1}{\xi} \ln ^{\kappa \frac{n+2}{n}}(M \xi) d \xi=\int_{\ln M}^{\ln M t} y^{\kappa \frac{n+2}{n}} d y=\frac{1}{1+\kappa \frac{n+2}{n}}\left[\ln ^{1+\kappa \frac{n+2}{n}} M t-\ln ^{1+\kappa \frac{n+2}{n}} M\right]
$$

if $t>1 \geq \frac{2}{M}$. In short,

$$
\mathcal{L}(t)=c_{1} \ln ^{\gamma} M t-c_{2}, \quad \text { for all } t>1
$$

where

$$
\gamma:=1+\kappa \frac{n+2}{n}
$$

and $c_{1}:=\frac{1}{\gamma}$, and $c_{2}:=\frac{1}{\gamma} \ln ^{\gamma} M$. Accordingly, we have

$$
\mathcal{L}^{-1}(t)=\frac{1}{M} \exp \left(\left(\frac{t+c_{2}}{c_{1}}\right)^{\frac{1}{\gamma}}\right) \quad \text { for all } t \geq c_{1} \ln ^{\gamma} M-c_{2},
$$

and

$$
\left(\mathcal{L}^{-1}\right)^{\prime}(t)=\frac{1}{M} \exp \left(\frac{t+c_{2}}{c_{1}}\right)^{\frac{1}{\gamma}} \frac{1}{\gamma}\left(\frac{t+c_{2}}{c_{1}}\right)^{\frac{1}{\gamma}-1} \frac{1}{c_{1}} \quad \text { for all } t>c_{1} \ln ^{\gamma} M-c_{2}
$$

that is

$$
\ln \left(\left(\mathcal{L}^{-1}\right)^{\prime}(t)\right)=\left(\frac{t+c_{2}}{c_{1}}\right)^{\frac{1}{\gamma}}+\left(\frac{1}{\gamma}-1\right) \ln \left(\frac{t+c_{2}}{c_{1}}\right)-\ln M
$$

if $t>c_{1} \ln ^{\gamma} M-c_{2}$, and thus there are positive constants $c_{5}, c_{6}$, and $t_{0}>0$ such that

$$
c_{5} t^{\frac{1}{\gamma}} \leq \ln \left(\mathcal{L}^{-1}\right)^{\prime}(t) \leq c_{6} t^{\frac{1}{\gamma}} \quad \text { for all } t>t_{0} .
$$

Having derived properties of $\ell$ for general parameters, we can now turn our attention to the proof of part i) of Corollary 1.6. Namely, fixing $R_{\star}>0, \beta>0, c_{0}>0$ and $\alpha \in(0,1)$ such that $u_{0}(x) \geq c_{0} e^{-\alpha|x|^{\beta}}$ for all $x \in \mathbb{R}^{n} \backslash B_{R_{\star}}$, we take any $q \in(\alpha, 1)$ and $M \geq 2$ and let $\kappa:=\frac{n}{\beta}>0$. By possibly enlarging $R_{\star}$ we ensure that $c_{0} M^{-q} \geq e^{-(q-\alpha)|x|^{\beta}}$ for all $x \in \mathbb{R}^{n} \backslash B_{R_{\star}}$, so that

$$
u_{0}(x) \geq c_{0} e^{-\alpha|x|^{-\beta}} \geq M^{q} e^{-q|x|^{\beta}}=\left[\ell^{-1}\left(\frac{1}{|x|^{n}}\right)\right]^{q} \quad \text { for all } x \in \mathbb{R}^{n} \backslash B_{R_{\star}} .
$$

In light of the attributes of $\ell$ previously asserted, from Theorem 1.5 and (6.21) we obtain $t_{0}>0$, $C_{1}>0$, and $C_{2} \geq 0$ such that $\mathcal{E}(t) \leq C_{1} t^{\frac{1}{\gamma}}+C_{2}$ for $t>t_{0}$, which in turn yields

$$
\mathcal{E}(t) \leq C t^{\frac{1}{1+\frac{n+2}{\beta}}} \quad \text { for } t>t_{0},
$$


if we set $C:=C_{1}+C_{2} t_{0}^{-\frac{1}{\gamma}}$ and take into account (6.20).

In order to prove Corollary 1.6 ii), we fix $\alpha>0, \beta>0$ and $C_{0}>0$ such that $u_{0}(x) \leq C_{0} e^{-\alpha|x|^{\beta}}$ for all $x \in \mathbb{R}^{n}$, and pick $\varepsilon>0$. We choose $M>\max \left\{2 C_{0}, 2\right\}$ and let $\kappa:=\frac{n}{\beta}+\frac{n \varepsilon}{n+2}$. These choices entail that, since $\xi_{2}=\frac{M}{2}>1$, for any $q_{0} \in(0,1)$ we have

$$
u_{0}(x) \leq \bar{u}_{0}(x):=C_{0} e^{-\alpha|x|^{\beta}} \leq C_{0} \leq \frac{M}{2}=\xi_{2}<\xi_{2}^{\frac{2}{1+q_{0}}}<\xi_{2}^{2} \quad \text { for all } x \in \mathbb{R}^{n},
$$

and that $\kappa \beta>n$, so that

$$
\int_{\mathbb{R}^{n}} \ell(\bar{u}(x)) d x=\int_{\mathbb{R}^{n}} \ell\left(C_{0} e^{-\alpha|x|^{\beta}}\right) d x=\int_{\mathbb{R}^{n}} \ln ^{-\kappa}\left(\frac{M}{C_{0}} e^{\alpha|x|^{\beta}}\right) d x=\int_{\mathbb{R}^{n}} \frac{1}{\left(\ln \frac{M}{C_{0}}+\alpha|x|^{\beta}\right)^{\kappa}} d x<\infty .
$$

Furthermore, according to (6.20),$\gamma=1+\frac{n+2}{\beta}+\varepsilon$. Consequently, Theorem 1.5 becomes applicable and due to (6.21) directly results in Corollary 1.6 ii).

\subsubsection{Doubly exponentially decaying data. Proof of Corollary 1.7}

Proof of Corollary [1.7. Fixing $\beta>0, \alpha>0, C_{0}>0$ and $\varepsilon>0$ as in the assumptions of Corollary 1.7. we first pick $B<\beta$ such that $\frac{n+2}{B}<\frac{n+2}{\beta}+\varepsilon$ and choose $A>1$ and $\widetilde{C}_{0}>C_{0}$ such that $C_{0} e^{-\alpha|x|^{\beta}} \leq \widetilde{C}_{0} e^{-A|x|^{B}}$ for all $x \in \mathbb{R}^{n}$. Moreover, we define $\gamma:=\frac{n+2}{\beta}+\varepsilon$, let $M>\max \left\{\widetilde{C}_{0} e e^{-A}, e, \widetilde{C}_{0}\right\}$ and choose $\xi_{2} \in\left[\max \left\{\widetilde{C}_{0} e^{-A}, 1\right\}, \frac{M}{e}\right)$. Then

$$
u_{0}(x) \leq \bar{u}_{0}(x):=\widetilde{C}_{0} e^{-A e^{|x|^{B}}} \leq \widetilde{C}_{0} e^{-A}<\xi_{2}<\xi_{2}^{\frac{2}{1+q_{0}}}<\xi_{2}^{2} \quad \text { for all } x \in \mathbb{R}^{n}
$$

for arbitrary $q_{0} \in(0,1)$. Moreover, we let $\kappa:=\frac{n \gamma}{n+2}$ and define

$$
\ell(\xi)= \begin{cases}0, & \xi=0, \\ \ln ^{-\kappa} \ln \left(\frac{M}{\xi}\right), & \xi \in\left(0, \xi_{2}\right), \\ \ln ^{-\kappa} \ln \left(\frac{M}{\xi_{2}}\right), & \xi \geq \xi_{2} .\end{cases}
$$

Then

$$
\int_{\mathbb{R}^{n}} \ell\left(\bar{u}_{0}(x)\right) d x=\int_{\mathbb{R}^{n}} \ln ^{-\kappa} \ln \left(\frac{M}{\widetilde{C}_{0}} e^{A e^{|x|^{B}}}\right) d x \leq \int_{\mathbb{R}^{n}} \frac{1}{\left(\ln A+|x|^{B}\right)^{\kappa}} d x
$$

is finite, because $\kappa B=\frac{B \gamma}{n+2} \cdot n>n$. Furthermore, (1.17) and $\ell \in C^{0}([0, \infty))$ are apparently satisfied and $\ell$ is strictly increasing on $\left(0, \xi_{2}\right)$. In addition, (1.22) and (1.23) have been shown in [8, Lemma 3.11]. By [8, Lemma 2.1], this also implies (1.21). Moreover, (1.18) holds, because $\xi \mapsto \xi \ln ^{-\kappa \frac{n+2}{n}} \ln M \xi$ is nondecreasing for large values of $\xi$ (as can easily be seen by checking the sign of the derivative $\left.\ln ^{-\kappa \frac{n+2}{n}-1} \ln M \xi \cdot\left(\ln \ln M \xi-\frac{\kappa(n+2)}{n \ln M \xi}\right)\right)$. Also (1.19) holds true, because

$$
\int_{1}^{\infty} \frac{1}{\xi} \ell^{-\frac{n+2}{n}}\left(\frac{1}{\xi}\right) d \xi=\int_{1}^{\infty} \frac{1}{\xi} \ln \frac{\kappa+2}{n} \ln M \xi d \xi=\int_{\ln M}^{\infty} \ln ^{\kappa \frac{n+2}{n}} y d y=\infty
$$


The inverse $\ell^{-1}:\left[0, \ln ^{-\kappa} \ln \frac{M}{\xi_{2}}\right) \rightarrow\left[0, \xi_{2}\right)$ is given by $\ell^{-1}(\xi)=M e^{-e^{\xi^{-\frac{1}{\kappa}}}}$ for $\xi \in\left(0, \ln ^{-\kappa} \ln \frac{M}{\xi_{2}}\right)$. In this case it is more difficult to give an explicit expression for $\mathcal{L}^{-1}$ than before. We provide a workaround in the following: Since $\gamma=\kappa \frac{n+2}{n}$, the function $\mathcal{L}$ is given by

$$
\mathcal{L}(t)=\int_{\ln M}^{\ln M+\ln t} \ln ^{\gamma} y d y \quad \text { for all } t>1 .
$$

We employ positivity and monotonicity of $\ln ^{\gamma}$, and thereby may infer

$$
\mathcal{L}(t) \leq \int_{1}^{2 \ln t} \ln ^{\gamma} y d y \leq 2 \ln t \ln ^{\gamma}(2 \ln t) \leq 2 \ln t(\ln 2+\ln \ln t)^{\gamma} \leq 2 \ln t(2 \ln \ln t)^{\gamma} \leq 2^{1+\gamma} \ln t \ln ^{\gamma} \ln t
$$

as long as $\ln t>\ln M$ and $\ln \ln t \geq \ln 2$. Inserting $\mathcal{L}^{-1}(t)$ istead of $t$ and using $\mathcal{L}\left(\mathcal{L}^{-1}(t)\right)=t$, we obtain that

$$
t \leq 2^{1+\gamma} \ln \mathcal{L}^{-1}(t) \ln ^{\gamma} \ln \mathcal{L}^{-1}(t)
$$

for sufficiently large $t$ and therefore

$$
e^{f(t)}(f(t))^{\gamma} \geq 2^{-1-\gamma} t \quad \text { for all } t>t_{0},
$$

with some $t_{0}>0$ and if we abbreviate $f(t):=\ln \ln \mathcal{L}^{-1}(t)$.

We recall that the Lambert $W$ function $W$ (see [4]) is defined to be the inverse of $0 \leq x \mapsto x e^{x}$. An inverse of $0 \leq x \mapsto x^{\gamma} e^{x}$ is given by $0 \leq x \mapsto \gamma W\left(\frac{1}{\gamma} x^{\frac{1}{\gamma}}\right)$ and we see from (6.22) that, given any $c>0$, there are $t_{0}>0$ and $c_{1}>0$ such that

$$
f(c t) \geq \gamma W\left(\frac{1}{\gamma}\left(c_{1} t\right)^{\frac{1}{\gamma}}\right) \quad \text { for all } t>t_{0} .
$$

By taking the logarithm on both sides of the defining relation for $W$, namely in $W(z) e^{W(z)}=z$ for $z>0$, we obtain

$$
W(z)+\ln W(z)=\ln z \quad \text { for } z>0
$$

and since $W(z)>1$ for $z>e$, this entails $W(z)<\ln z$ for $z>e$. We can use this estimate in (6.24) to infer that

$$
W(z)=\ln z-\ln W(z)>\ln z-\ln \ln z \quad \text { for all } z>e .
$$

Employing this together with (6.23), we can find positive constants $c_{2}, c_{3}, c_{4}$, and $t_{1}>0$, such that

$$
\begin{aligned}
f(c t) & \geq \gamma W\left(\left(c_{2} t\right)^{\frac{1}{\gamma}}\right) \geq \gamma \ln \left(\left(c_{2} t\right)^{\frac{1}{\gamma}}\right)-\gamma \ln \left(\ln \left(\left(c_{2} t\right)^{\frac{1}{\gamma}}\right)\right) \\
& =\ln \left(c_{2} t \ln ^{-\gamma}\left(\left(c_{2} t\right)^{\frac{1}{\gamma}}\right)\right)=\ln \left(c_{3} t \ln ^{-\gamma}\left(c_{2} t\right)\right) \\
& =\ln \left(c_{3} t\left(\ln c_{2}+\ln t\right)^{-\gamma}\right) \geq \ln \left(c_{4} t \ln ^{-\gamma} t\right) \quad \text { for all } t>t_{1} .
\end{aligned}
$$

Accordingly,

$$
\mathcal{L}^{-1}(c t)=e^{e^{f(c t)}} \geq e^{e^{\ln \left(c_{4} t \ln ^{-\gamma} t\right)}}=e^{c_{4} t \ln ^{-\gamma} t} \quad \text { for } t>t_{1} .
$$


Furthermore, there is $\xi_{0}>0$ such that

$$
\mathcal{L}^{\prime}(\xi)=\frac{1}{\xi} \ln ^{\kappa \frac{n+2}{n}} \ln M \xi \leq \frac{1}{\sqrt{\xi}} \quad \text { for all } \xi>\xi_{0} .
$$

If we combine (6.25) and (6.26) with the monotonicity of $\frac{1}{\mathcal{L}^{\prime}}$ guaranteed by (1.18), we can find $t_{2}>0$ such that

$$
\left(\mathcal{L}^{-1}\right)^{\prime}(c t)=\frac{1}{\mathcal{L}^{\prime}\left(\mathcal{L}^{-1}(t)\right)} \geq \frac{1}{\mathcal{L}^{\prime}\left(e^{c_{4} t \ln ^{-\gamma} t}\right)} \geq e^{\frac{c_{4}}{2} t \ln ^{-\gamma} t} \quad \text { for all } t>t_{2} .
$$

In conclusion, together with Theorem 1.5 ii) this proves Corollary 1.7.

\section{References}

[1] Aronson, D.G.: The porous medium equation. Lecture Notes in Mathematics Vol. 1224: Nonlinear diffusion problems. Springer, Berlin etc., 1-46 (1986)

[2] Bertsch, M., Dal Passo, R., Ughi, M.: Discontinuous "viscosity" solutions of a degenerate parabolic equation. Trans. Amer. Math. Soc. 320 (2), 779-798 (1990)

[3] Bomze, I.M.: Dynamical aspects of evolutionary stability. Monatsh. Math. 110, 189-206 (1990)

[4] Corless, R. M., Gonnet, G. H., Hare, D. E. G., Jeffrey, D. J., Knuth, D. E.: On the Lambert $W$ function. Adv. Comput. Math. 5, 329-359 (1996)

[5] Fila, M., King, J.R., Winkler, M., Yanagida, E.: Linear Behaviour of Solutions of a Superlinear Heat Equation. J. Math. Anal. Appl. 340, 401-409 (2008)

[6] Fila, M., Vázquez, J.L., Winkler, M., Yanagida, E.: Rate of Convergence to Barenblatt Profiles for the Fast Diffusion Equation. Arch. Rat. Mech. Anal. 204, 599-625 (2012)

[7] Fila, M., Winkler, M.: Slow growth of solutions of superfast diffusion equations with unbounded initial data. J. London Math. Soc. 95, 659-683 (2017)

[8] Fila, M., Winkler, M.: A Gagliardo-Nirenberg-type inequality and its applications to decay estimates for solutions of a degenerate parabolic equation. Preprint

[9] Kavallaris, N., Lankeit, J., Winkler, M.: On a degenerate non-local parabolic problem describing infinite dimensional replicator dynamics. SIAM J. Math. Anal. 49 (2), 954-983 (2017)

[10] Kravvaritis, D., Papanicolaou, V.G., Yannacopoulos, A.N.: Similarity solutions for a replicator dynamics equation. Ind. Univ. Math. Journal 57, 1929-1946 (2008)

[11] Kravvaritis, D., Papanicolaou, V.G., Xepapadeas, A., Yannacopoulos, A.N.: On a class of operator equations arising in infinite dimensional replicator dynamics. Nonlin. Anal. RWA 11, 2537-2556 (2010)

[12] Kravvaritis, C., Papanicolaou: Singular equilibrium solutions for a replicator dynamics model. Elect. J. Diff. Equations 87, 1-8 (2011) 
[13] Ladyženskaja, O. A., Solonnikov, V. A., Ural'Ceva, N. N. Linear and Quasilinear Equations of Parabolic Type. AMS, Providence (1968)

[14] LankeIt, J.: Equilibration of unit mass solutions to a degenerate parabolic equation with a nonlocal gradient nonlinearity. Nonlin. Anal. TMA 135, 236-248 (2016)

[15] LeE, T.Y., NI, W.-M.: Global existence, large time behavior and life span of solutions of a semilinear parabolic Cauchy problem. Trans. Amer. Math. Soc. 333, 365-378 (1992)

[16] Luckhaus, S., Dal Passo, R.: A Degenerate Diffusion Problem Not in Divergence Form. J. Diff. Eqns. 69, 1-14 (1987)

[17] Maynard Smith, J.: Evolution and The Theory of Games. Cambridge Univ. Press (1982)

[18] Oechssler, J., Riedel, F.: Evolutionary dynamics on infinite strategy spaces. Econom. Theory 17, 141-162 (2001)

[19] Papanicolaou, V.G., Smyrlis, G.: Similarity solutions for a multi-dimensional replicator dynamics equation. Nonlin. Anal. TMA, 71, 3185-3196 (2009)

[20] Papanicolaou, V.G., Vasilakopoulou, K.: Similarity solutions of a replicator dynamics equation associated to a continuum of pure strategies. Electron. J. Diff. Equations, 10, 1-16 (2015)

[21] Quittner, P., Souplet, Ph.: Superlinear Parabolic Problems. Blow-up, Global Existence and Steady States. Birkhäuser Advanced Texts, Basel/Boston/Berlin, 2007

[22] TAylor, P. D., Jonker, L. B.: Evolutionary stable strategies and game dynamics. Math. Biosci. 40, 145-156 (1978)

[23] VÁzquez, J.L.: Smoothing and Decay Estimates for Nonlinear Diffusion Equations. Equations of Porous Medium Type. Oxford Lecture Series in Mathematics and Its Applications 33, Oxford University Press, 2006

[24] Wiegner, M.: A Degenerate Diffusion Equation with a Nonlinear Source Term. Nonlin. Anal. TMA 28, 1977-1995 (1997)

[25] Winkler, M.: On the Cauchy Problem for a Degenerate Parabolic Equation. Z. Anal. Anwend. 20, 677-690 (2001)

[26] WinkleR, M.: Oscillating solutions and large $\omega$-limit sets in a degenerate parabolic equation. J. Dyn. Differ. Eq. 20 (1), 87-113 (2008)

[27] Winkler, M.: Slowly traveling waves and homoclinic orbits in a nonlinear parabolic equation of super-fast diffusion type. Math. Ann. 355 (2), 519-549 (2013) 